\title{
On a Mathematical Theory of Coded Exposure
}

\author{
Yohann Tendero* $\quad$ Stanley Osher*
}

\begin{abstract}
This paper proposes a mathematical model and formalism to study coded exposure (flutter shutter) cameras. The model includes the Poisson photon (shot) noise as well as any additive (readout) noise of finite variance. This is an improvement compared to our previous work that only considered the Poisson noise. In addition, closed formulae for the Mean Square Error and Signal to Noise Ratio of the coded exposure method are given. These formulae take into account for the whole imaging chain, i.e., the Poisson photon (shot) noise, any readout noise of finite variance as well as the deconvolution and are valid for any exposure code. In addition, we give an explicit formula that gives an absolute upper bound for the gain of any coded exposure cameras in function of the temporal sampling of the exposure code. The gain is to be understood in terms of Mean Square Error (or equivalently in terms of Signal to Noise Ratio), with respect to a snapshot.
\end{abstract}

Keywords: Coded exposure, computational photography, flutter shutter, motion blur, mean square error (MSE), signal to noise ratio (SNR).

\section{Introduction}

Since the founding papers [2, 3, 4, 37, 38, 39] of Agrawal, Raskar et al. coded exposure (flutter shutter) method has received at lot follow ups $[1,11,12,14,16,17,18,19,27,28,29$, $30,31,32,33,34,35,36,40,42,43,46,47,48,50,51,52,53,54]$. In a nutshell, the authors proposed to open and close the camera shutter, according to a sequence called "code", during the exposure time. By this clever exposure technique, the coded exposure method permits one to arbitrarily increase the exposure time when photographing (flat) scenes moving at a constant velocity. Note that with a coded exposure method only one picture is stored/transmitted. A rich body of empirical results that suggest that the coded exposure method allows for a gain in terms of Mean Square Error (MSE) or Signal to Noise Ratio (SNR) compared to a classic camera, i.e., a snapshot. Therefore, the coded exposure method seems to be a magic tool that should equip all cameras. However, to the best of our knowledge, little is known on the coded exposure method from a rigorous mathematical point of view. Hence, it seems crucial for the applications to build a theory able to shed some light on this coded exposure method. For instance, to the best of our knowledge, little is know on the gain, in terms of MSE or SNR, of this coded exposure method compared to a standard (snapshot) camera. This papers proposes a mathematical model of photon acquisition by a light sensor. The model can cope with any additive readout noise of finite variance in addition to the Poisson photon (shot) noise. The model is compatible with the Shannon-Whittaker framework, assumes that the relative camera

\footnotetext{
*Department of Mathematics - University of California Los Angeles 520 Portola Plaza, Los Angeles, CA 90095, United States, tendero@math.ucla.edu. Questions, comments, or corrections to this document may be directed to that email address. Yohann Tendero and Stanley Osher are supported on the grant ONR No: N000141410683.
} 


\section{Report Documentation Page}

Form Approved

OMB No. 0704-0188

Public reporting burden for the collection of information is estimated to average 1 hour per response, including the time for reviewing instructions, searching existing data sources, gathering and maintaining the data needed, and completing and reviewing the collection of information. Send comments regarding this burden estimate or any other aspect of this collection of information,

including suggestions for reducing this burden, to Washington Headquarters Services, Directorate for Information Operations and Reports, 1215 Jefferson Davis Highway, Suite 1204, Arlington

VA 22202-4302. Respondents should be aware that notwithstanding any other provision of law, no person shall be subject to a penalty for failing to comply with a collection of information if it

does not display a currently valid OMB control number.

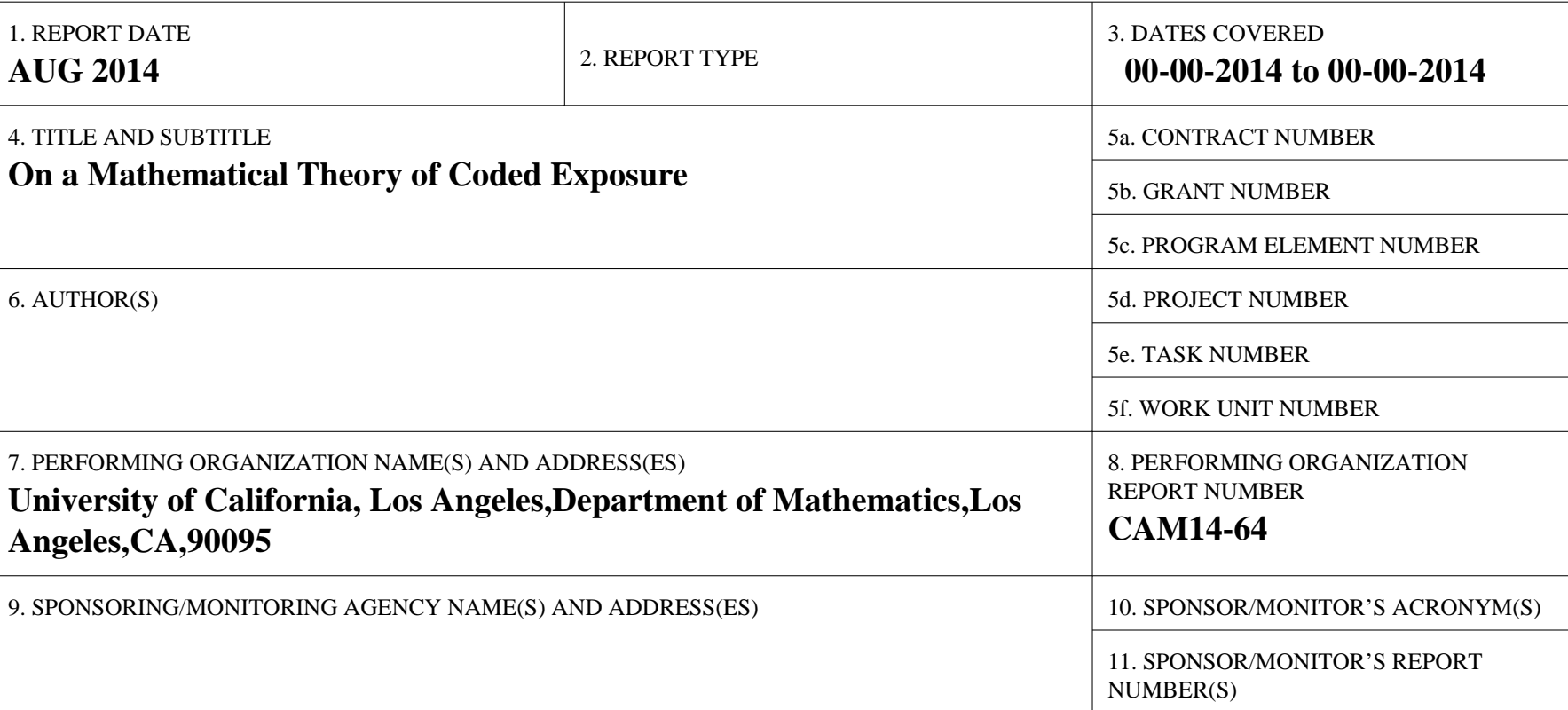

12. DISTRIBUTION/AVAILABILITY STATEMENT

Approved for public release; distribution unlimited

13. SUPPLEMENTARY NOTES

14. ABSTRACT

This paper proposes a mathematical model and formalism to study coded exposure (flutter shutter)

cameras. The model includes the Poisson photon (shot) noise as well as any additive (readout) noise of finite variance. This is an improvement compared to our previous work that only considered the Poisson noise. In addition, closed formulae for the Mean Square Error and Signal to Noise Ratio of the coded exposure method are given. These formulae take into account for the whole imaging chain, i.e., the Poisson photon (shot) noise, any readout noise of finite variance as well as the deconvolution and are valid for any exposure code. In addition, we give an explicit formula that gives an absolute upper bound for the gain of any coded exposure cameras in function of the temporal sampling of the exposure code. The gain is to be understood in terms of Mean Square Error (or equivalently in terms of Signal to Noise Ratio), with respect to a snapshot.

\begin{tabular}{|c|c|c|c|c|c|}
\hline \multicolumn{3}{|c|}{ 16. SECURITY CLASSIFICATION OF: } & \multirow{2}{*}{$\begin{array}{l}\text { 17. LIMITATION OF } \\
\text { ABSTRACT } \\
\text { Same as } \\
\text { Report (SAR) }\end{array}$} & \multirow{2}{*}{$\begin{array}{c}\text { 18. NUMBER } \\
\text { OF PAGES } \\
\mathbf{4 4}\end{array}$} & \multirow{2}{*}{$\begin{array}{l}\text { 19a. NAME OF } \\
\text { RESPONSIBLE PERSON }\end{array}$} \\
\hline $\begin{array}{c}\text { a. REPORT } \\
\text { unclassified }\end{array}$ & $\begin{array}{c}\text { b. ABSTRACT } \\
\text { unclassified }\end{array}$ & $\begin{array}{c}\text { c. THIS PAGE } \\
\text { unclassified }\end{array}$ & & & \\
\hline
\end{tabular}


scene velocity is constant and known, that the sensor does not saturate, that the quantization effects can be neglected, that the readout noise has finite variance and that the coded exposure method allows for an invertible transformation among the class of band limited functions (this means that the observed image can be deblurred using a filter). Note that with this model the image has a structure: the image is assumed to be band limited.

To be thorough, a mathematical analysis of a camera requires to go rigorously from the continuous observed scene to the discrete samples of the final restored image. This is needed to mathematically analyze the whole image chain: from the photon emission to the final restored image via the observed discrete samples measured by the camera. To the best of our knowledge, the coded exposure method is very useful for moving scenes. Consequently, we need a formalism capable of dealing with moving scenes. Since the observed scene moves continuously with respect to the time we adopt a continuous point of view. This means that we shall model the observed scene as a function $s$. Loosely speaking, $s(x)$ give the light intensity at a spatial position $x$. (By opposition, a discrete formalism would model the observed scene as a vector of $\mathbb{R}^{n}$ but requires a more restrictive assumption, see below.) We shall rely on the Shannon-Whittaker framework (see, e.g, [15]) to perform the mathematical analysis of sampling-related questions. This framework requires the structure of band limited (with a cut off frequency) signals or images and will allow us to perform a rigorous mathematical analysis of the coded exposure method. Recall that a discrete formalism would model the observed scene as a vector of $\mathbb{R}^{n}$ and the convolution would use Toepliz matrices. Therefore, the scene would be assumed to be periodic and also band limited for sampling purposes. Note that the continuous formalism that we shall develop in this paper does not require to assume that the observed scene $s$ is periodic. (This is needed because, to the best of our knowledge, most natural scenes are not periodic.) However, the adaptation of the formalism that we shall develop in this paper to periodic band limited scene is straightforward if needed for some application.

Our first goal is to provide closed mathematical formulae that give the MSE and SNR of images obtained by a coded exposure camera. Therefore, we shall start by carefully model the photon acquisition by a light sensor then deduce a mathematical model of the coded exposure method. The mathematical model of camera that we shall develop in this paper has not, to the best of our knowledge, been developed in the existing literature on the coded exposure method. Indeed, the model we shall develop in this paper is able to cope with the Poisson photon (shot) noise in addition to any additive (sensor readout) noise of finite variance and does not require to assume that the observed scene is periodic. For example, the model developed in [48] does not consider any additive (sensor readout) noise. The formulae that give the MSE and SNR of the final crisp image

1. Assumes the Shannon-Whittaker framework that i) requires band limited (with a frequency cut off) images, and that ii) the pixel size is designed according to the ShannonWhittaker theory.

2. Assumes that the relative camera scene velocity is constant and known.

3. Assumes that the sensor does not saturate.

4. Assumes that there is no quantization.

5. Assumes that the additive (sensor readout) noise has zero mean and finite variance.

6. Assumes that the coded exposure allows for an invertible transformation among the class of band limited functions. (This means that the observed image can be deblurred using a filter.) 
7. Neglects the boundaries effects for the deconvolution. (This is in favor of the coded exposure because the inverse filter of a coded exposure camera has larger support than the inverse filter of a snapshot.)

To the best of our knowledge, a sensor readout (additive) noise has zero mean. However, with our formalism, the adaptation to non zero mean additive (sensor readout) noise is straightforward if needed for some application.

This paper proposes a mathematical model of coded exposure cameras that includes any zero mean additive (sensor readout) noise of finite variance in addition to the Poisson photon (shot) noise. This model allows us to prove a theorem that gives closed formulae for the MSE and SNR of coded exposure cameras (see theorem 3.8 page 23). To the best of our knowledge, the formula that gives the SNR of the coded exposure method, cannot be found in the existing literature on the coded exposure method. We also give an upper bound for the gain of the coded exposure method, in terms of MSE and SNR (see corollary 4.2 page 31), with respect to a snapshot in function of the temporal frequency sampling of the exposure code. To the best of our knowledge, the result stated in corollary 4.2 cannot be found in the existing literature. The bound is applicable, to the best of our knowledge, to every existing exposure code of the literature.

The upper bound of corollary 4.2 page 31 is illustrated on figure 2 page 31 . In addition, table 1 page 33 provides numerical experiments illustrating these results.

The paper is organized as follows. Section 2 gives a mathematical model classic cameras. This mathematical model is extended in section 3 to model coded exposure cameras. Section 4 gives an upper bound for the gain of the coded exposure method, in terms of MSE and SNR with respect to a snapshot, in function of the temporal sampling of the code. The annexes AG contain several proofs of propositions that are used throughout this paper. A glossary of notations is in annex $\mathrm{H}$ page 40. (In the sequel latin numerals refer to the glossary of notations page 40.)

\section{A mathematical model of classic cameras}

The goal of this section is to provide a mathematical model of the photon acquisition by a light sensor and the formalism that we shall use in the sequel.

As usual in the coded exposure literature $[3,4,5,12,33,34,48,54]$ and for the sake of the clarity we shall formalize the coded exposure method using a one dimensional framework. In other words, the sensor array and the observed image are assumed be one dimensional. This one dimensional framework is no limitation. Indeed, as we have seen, we assume that the image acquisition obeys the Shannon-Whittaker sampling theory. This means that the frequency cut off is compatible with the image grid sampling. The extension to any two dimensional grid (and two dimensional images) is straightforward. The sketch of the proof is in annex A page 33. Therefore, the one dimensional framework that we shall consider is no limitation for the scope of this paper that proposes a mathematical analysis of coded exposure cameras. A fortiori, the calculations of MSE and SNR that we shall propose in this paper remain valid for two dimensional images. In addition, we will assume that the motion blur kernel is known, i.e., the relative camera-scene velocity vector and the exposure code (or function) are known (this kernel is called "PSF motion" in, e.g., [2]).

We now turn to the mathematical model of photons acquisition by a light sensor. 


\subsection{A mathematical model of photons acquisition by a light sensor that includes any additive (sensor readout) noise of finite variance in addition to the Poisson photon (shot) noise}

The goal of this subsection is to give a rigorous mathematical definition (see definition 2.1 page 8 ) of the samples produced by a pixel sensor that observes a moving scene. This definition of the observed sample can cope with any additive zero mean (sensor readout) noise of finite variance in addition to the standard Poisson photon (shot) noise. Note that the model developed in [48] do not consider any additive (sensor readout) noise. Therefore, the results of [48] do not include this more elaborated mathematical model. In particular, the advantages of the coded exposure method in terms of MSE, with this more elaborated set up, are, to the best of our knowledge, open questions. Note that, to the best of our knowledge, the optimization of a coded exposure camera is an open question.

We consider a continuous formalism in order to ease the transition from steady scenes to scenes moving at an arbitrary real velocity. Another advantage of this continuous formalism is that it allows us to avoid the implicit periodic assumption of the observed scene needed if one uses Toeplitz matrices to represent the convolutions. (This is needed because, to the best of our knowledge, most natural scenes are not periodic.)

We now sketch the construction of our camera model. We first consider the photon emission, then include the optical and sensor kernels, then include the effect of the exposure time and of the motion to our model. The lasts two steps consists in adding the Poisson photon (shot) noise and the additive (sensor readout) noise to our camera model. The camera model that we shall consider in this paper is depicted in figure 1.

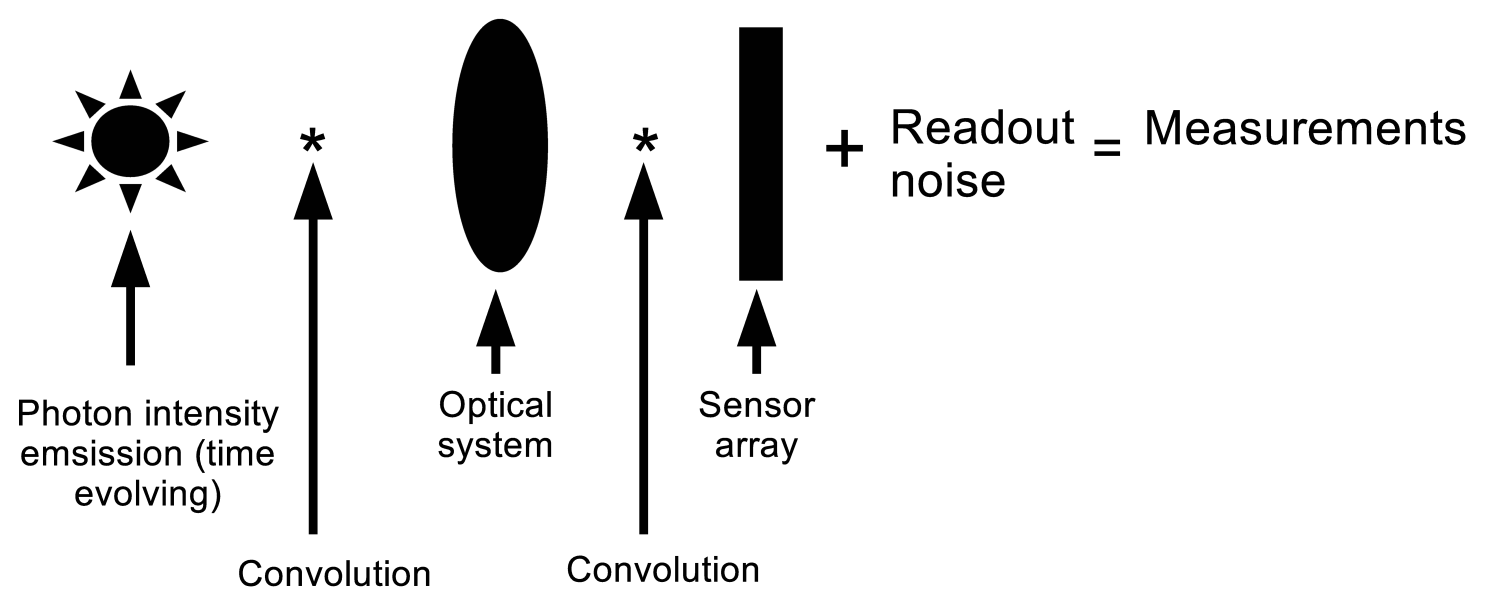

Figure 1: Schematic diagram of our camera model. The observed scene emits light and moves at velocity $v$. The light undergoes the blur of the optical system and is measured by a pixel sensor. The pixel sensor produces a Poisson random variable (shot noise) that is further corrupted by an additive (sensor readout) noise of finite variance to produce the observed sample.

We assume that the observed scene emits photons at a deterministic rate $s$ defined by

$$
\begin{aligned}
s: \mathbb{R} & \longrightarrow(0,+\infty) \\
x & \longmapsto s(x) .
\end{aligned}
$$

Here and in the sequel, the variable $x$ represents the spatial position. (We will precise the unit of $x$, i.e., the unit we shall use to measure distances when we introduce the pixel sensor.) 
Intuitively, $s$ represents the ideal crisp image, i.e., the image that one would observed if there were no noise whatsoever, no motion, with a perfect optical system (formally the point spread function is a Dirac-mass) and the pixel sensor has an infinitesimal area. In a nutshell, $s(x)$ would be the grey level of the image at position $x$ in the idealistic case mentioned above. The quantity $s(x)$ can also be seen as the intensity of light emission at $x$.

We now introduce the optical system in our model. The effect of the optical system is described by its point spread function (PSF) denoted $g$, and we assume that $g \geqslant 0$. Formally, the effect of the point spread function is modeled by a convolution in space (see, e.g., [24, equation 7.1 page 171] see also, e.g. [2, equation 1, section 2]). Therefore, in the noiseless case, if there is no motion, the grey level of the acquired image at position $x$ is, formally, described by

$$
(g * s)(x)
$$

where $*$ denotes the convolution (see $(\mathbf{x})$ for the definition). (Recall that here and in the rest of the text, Latin numerals refer to the formulae in the final glossary page 40.) We will give the assumptions on $g$ and $s$ so that the quantity in (1) is well defined later on.

A pixel sensor can be small but has nevertheless a positive area. Indeed, a pixel sensor integrates the incoming light $g * s$ (the scene is observed through the optical system) on some surface element of the form $\left[x_{1}, x_{2}\right] \subset \mathbb{R}$ with $x_{1}<x_{2}$. Therefore, formally, the output of a pixel sensor supported by $\left[x_{1}, x_{2}\right]$, in the noiseless case and without motion, is

$$
\int_{x_{1}}^{x_{2}}(g * s)(y) d y
$$

In the sequel we shall assume that all the pixel sensors of the sensor array have the same length. Mathematically, we can normalize this length so that the all the pixel sensors of the array have unit length. This correspond to using the pixel sensor length as unit to measure distances. Thus, this represents no limitation. Hence, from now on the unit of $x$ is the pixel sensor length. By definition, with this unit, all the pixel sensor have lengths 1. Therefore, from now on when we speak of a pixel sensor centered at $x$ we mean that the pixel sensor is supported on the interval $\left[x-\frac{1}{2}, x+\frac{1}{2}\right]$.

Hence, from (2) we deduce that the output of a pixel sensor supported on the interval $\left[x-\frac{1}{2}, x+\frac{1}{2}\right]$, that stares at the scene $s$ through the optical system modeled by $g$, is, in the noiseless case and without motion,

$$
\int_{x-\frac{1}{2}}^{x+\frac{1}{2}}(g * s)(y) d y=(\underbrace{\mathbb{1}_{\left[-\frac{1}{2}, \frac{1}{2}\right]} * g * s}_{u})(x) .
$$

Consider the deterministic function formally defined by $u:=\mathbb{1}_{\left[-\frac{1}{2}, \frac{1}{2}\right]} * g * s$. The deterministic quantity $u(x)$ represents the grey level of the image at position $x$ if there were no noise and no motion. Indeed, $u$ contains the kernels of the optical system $g$ and of the sensor. Note that the quantity $u(x)$ can also be seen as an intensity of light emission received by a unit pixel sensor centered at $x$. With the formalism of, e.g., $[2$, equation 1 , section 2$] \mathbb{1}_{\left[-\frac{1}{2}, \frac{1}{2}\right]}$ represents " $h_{\text {sensor }}$ " and $g$ represents " $h_{\text {lens" }}$ ".

We now introduce the exposure time in our model. Indeed, the sensor accumulates the light during a time span of the form $\left[t_{1}, t_{2}\right] \subset \mathbb{R}$, with $t_{1}<t_{2}$. We denote by $\Delta t$ the positive quantity $\Delta t:=t_{2}-t_{1}$ called exposure time. Thus, from (3), the output of a pixel sensor centered at $x$ that integrates on the time interval $\left[t_{1}, t_{2}\right]$ is, in the noiseless case

$$
\int_{t_{1}}^{t_{2}} \int_{x-\frac{1}{2}}^{x+\frac{1}{2}}(g * s)(y) d y d t=\int_{t_{1}}^{t_{2}} u(y) d t .
$$


Note that the quantity in (4) is the amount of light measured by the pixel sensor, and it evolves linearly with the exposure time $\Delta t\left(=t_{2}-t_{1}\right)$.

We now extend the above formalism to cope with moving scenes. Without loss of generality (w.l.o.g.) we assume that the camera is steady while the scene $s$ moves. The coded exposure method permits to deal with uniform motions. Therefore, we assume that the scene $s$ moves at a constant velocity $v \in \mathbb{R}$ (measured in pixel per second) during the exposure time interval $\left[t_{1}, t_{2}\right]$. This means that the scene evolves with respect to the time as $s(x-v t)$. Here and in the sequel the temporal variable is denoted by $t$. Therefore, from (4) we deduce that the output of a pixel pixel sensor centered at $x$ and integrating on the time interval $\left[t_{1}, t_{2}\right]$ is, in the noiseless case,

$$
\int_{t_{1}}^{t_{2}} \int_{x-\frac{1}{2}}^{x+\frac{1}{2}}(g * s)(y-v t) d y d t=\int_{t_{1}}^{t_{2}} u(x-v t) d t .
$$

For example, suppose that we take a constant velocity $v=1$ in (5). In this case, the output of a pixel sensor centered at $x$ is, in the noiseless case,

$$
\int_{t_{1}}^{t_{2}} \int_{x-\frac{1}{2}}^{x+\frac{1}{2}}(g * s)(y-t) d y d t=\left(\mathbb{1}_{\left[t_{1}, t_{2}\right]} * \mathbb{1}_{\left[-\frac{1}{2}, \frac{1}{2}\right]} * g * s\right)(x)=\left(\mathbb{1}_{\left[t_{1}, t_{2}\right]} * u\right)(x),
$$

where $\mathbb{1}_{[a, b]}$ represents the characteristic function of the interval $[a, b]$. (See (3) for the last equality.) From this simple example we can qualitatively describe where the exposure code will act. Indeed, by a clever exposure technique, the coded exposure method will allow to replace the function $\mathbb{1}_{\left[t_{1}, t_{2}\right]}$ in the above formula by a more general class of functions that does not need to be window functions. With the formalism of, e.g., [2, equation 1, section 2] $\mathbb{1}_{\left[t_{1}, t_{2}\right]}$ represents " $h_{\text {motion". }}$

We now extend our model to cope with the Poisson photon (shot) noise and then will add the readout noise. The photon emission follows a Poisson distribution see, e.g., [7]. (If $X$ is a random variable that follows a Poisson distribution then all the possible realization of $X$ are in N. In addition, the probability of the event $X=k$ is $\mathbb{P}(X=k)=\frac{\lambda^{k} e^{-\lambda}}{k !}$, where $\lambda>0$ is the intensity of the Poisson random variable.) A pixel sensor is a photon counter. Indeed, a pixel sensor integrates the photons that are emitted by the moving observed scene $s$ on some surface element of the form $\left[x_{1}, x_{2}\right]$ on the time span $\left[t_{1}, t_{2}\right]$ and produces a sample. This sample follows a Poisson random variable. From (5), this means that the sample produced by a pixel sensor supported by $\left[x_{1}, x_{2}\right]$ and that integrates on the time span $\left[t_{1}, t_{2}\right]$ has law

$$
\mathcal{P}\left(\int_{t_{1}}^{t_{2}} \int_{x-\frac{1}{2}}^{x+\frac{1}{2}}(g * s)(y-v t) d y d t\right),
$$

where the notation $\mathcal{P}(\lambda)$ denotes a Poisson random variable of intensity $\lambda$. With (3) the above equation can be rewritten as

$$
\mathcal{P}\left(\int_{t_{1}}^{t_{2}} u(x-v t)\right)
$$

Thus, the value of this sample can be any realization of this Poisson random variable. Consequently, the probability that the sample has value $k \in \mathbb{N}$ when observing the scene $s$ on the time span $\left[t_{1}, t_{2}\right]$ with the pixel sensor centered at $x$ is

$$
\frac{\left(\int_{t_{1}}^{t_{2}} u(x-v t) d t\right)^{k} \exp \left(-\int_{t_{1}}^{t_{2}} u(x-v t) d y d t\right)}{k !} .
$$

This quantity is nothing but the probability that the pixel sensor counts $k$ photons during the time interval $\left[t_{1}, t_{2}\right]$. 
Consider the case where $v=0, t_{1}=0, t_{2}=\Delta t$ in (7). If the observed value $\operatorname{obs}(x)$ at a position $x \in \mathbb{R}$ follows $\mathcal{P}\left(\int_{0}^{\Delta t} u(x) d t\right)$ we have $\mathbb{E}(o b s(x))=\Delta t u(x)$. This means that, in expectation, the number of photon caught by the pixel sensor centered at $x$ increases linearly with the exposure time. If we time normalize the obtained quantity and consider, formally, a random variable $\mathfrak{u}_{e s t}(x)$ that follows $\frac{\mathcal{P}\left(\int_{0}^{\Delta t} u(x) d t\right)}{\Delta t}$ we obtain $\mathbb{E}\left(\mathfrak{u}_{e s t}(x)\right)=u(x)$. This means that $\mathbb{u}_{e s t}(x)$ estimates $u(x)$ without bias. In addition, we have $\operatorname{var}\left(\mathbb{u}_{e s t}(x)\right)=\frac{\Delta t u(x)}{\Delta t^{2}}=\frac{u(x)}{\Delta t}$. Consider the SNR on the space interval $[-R, R]$ given by $\frac{\frac{1}{2 R} \int_{-R}^{R} \mathbb{E}\left(\mathbb{u}_{e s t}(x)\right) d x}{\sqrt{\frac{1}{2 R} \int_{-R}^{R} \operatorname{var}\left(\mathbb{w}_{e s t}(x)\right) d x}}$. This definition of the SNR can be found in, e.g, [24, equation 1.39, p. 42], [5, equation 15 , p. 4], [3, equation 1, p. 2562]. We have

$$
\frac{\frac{1}{2 R} \int_{-R}^{R} \mathbb{E}\left(\mathbb{u}_{e s t}(x)\right) d x}{\sqrt{\frac{1}{2 R} \int_{-R}^{R} \operatorname{var}\left(\mathfrak{u}_{e s t}(x)\right) d x}}=\frac{\frac{1}{2 R} \int_{-R}^{R} u(x) d x}{\sqrt{\frac{1}{2 R} \int_{-R}^{R} \frac{u(x)}{\Delta t} d x}}=\frac{\sqrt{\frac{1}{2 R} \int_{-R}^{R} u(x) d x}}{\Delta t} .
$$

Therefore, assuming that $\mu$ the "mean signal level" [24, p. 42] ( $\mu$ relates to $\bar{i}_{0}$ in, e.g., [3, section 2])

$$
\mathbb{R}^{+} \ni \mu:=\lim _{R \rightarrow+\infty} \frac{1}{2 R} \int_{-R}^{R} u(x) d x
$$

is finite we can define the SNR by

$$
\operatorname{SNR}\left(\mathfrak{u}_{e s t}\right):=\frac{\lim _{R \rightarrow+\infty} \frac{1}{2 R} \int_{-R}^{R} \mathbb{E}\left(\mathfrak{w}_{e s t}(x)\right) d x}{\sqrt{\lim _{R \rightarrow+\infty} \frac{1}{2 R} \int_{-R}^{R} \operatorname{var}\left(\mathfrak{u}_{e s t}(x)\right) d x}} .
$$

Thus, we have $\operatorname{SNR}\left(\mathbb{u}_{e s t}\right)=\sqrt{\mu \Delta t}$. For example, if the mean photon emission $\mu$ doubles then the SNR is multiplied by a factor $\sqrt{2}$. (And we retrieve the fundamental theorem of photography.) Note that if we have no control over the photon emission then the only way to increase the SNR is to increase the exposure time $\Delta t$. Similarly, we define the MSE by

$$
\operatorname{MSE}\left(\mathbb{u}_{e s t}\right):=\lim _{R \rightarrow+\infty} \frac{1}{2 R} \int_{-R}^{R} \mathbb{E}\left(\left|\mathfrak{u}_{e s t}(x)-u(x)\right|^{2}\right) d x,
$$

whenever the limit exists, and we have $\operatorname{MSE}\left(\mathbb{u}_{e s t}\right)=\mu \Delta t$.

We are now in position to extend our model to include the additive (readout) noise. Here and in the sequel the additive (readout) noise of a pixel sensor centered at $x$ is modeled by a zero mean real random variable of finite variance denoted by $\eta(x)$. Therefore, from (6), the output of the pixel sensor centered at $x$ that integrates the photons on the time span $\left[t_{1}, t_{2}\right]$ can be, formally, any realization of the sum of the random variables

$$
\mathcal{P}\left(\int_{t_{1}}^{t_{2}} \int_{x-\frac{1}{2}}^{x+\frac{1}{2}}(g * s)(y-v t) d y d t\right)+\eta(x),
$$

or equivalently (see (3)),

$$
\mathcal{P}\left(\int_{t_{1}}^{t_{2}} u(x-v t) d t\right)+\eta(x)
$$

Recall that the deterministic quantity $u(x)$ represents the grey level of the image at position $x$ if there were no noise and no motion as it is seen by a pixel sensor centered at $x$. Indeed, recall that $u$ contains the kernels of the optical system $g$ and of the (normalized) pixel sensor. 
The quantity $\int_{t_{1}}^{t_{2}} u(x-v t) d t$ represents the amount of light received by a steady pixel sensor centered at $x$ that gathers the light emitted by the observed scene moving at velocity $v$ on the time interval $\left[t_{1}, t_{2}\right]$. Here and in the sequel, we call "observed sample" and denote $o b s(x)$ the sum of random variables formally defined in (9).

We now give a mathematical framework to make precise the above formulae. We assume that the scene $s \in L_{l o c}^{1}(\mathbb{R})$ so that the convolution in (3) page 5 is well defined. We will assume that the PSF furnishes a cut off frequency. This assumption is needed by the Shannon-Whittaker sampling theory. We will assume that the frequency cut off of $g$ is $\pi$, i.e., $g$ is $[-\pi, \pi]$ band limited. In other words, $\hat{g}(\xi)=0$ for any $\xi \in \mathbb{R}$ such that $|\xi|>\pi$, where, here and in the sequel, we denote by $\hat{g}$ or $\mathcal{F}(g)$ the Fourier transform of $g$ (see (xv) for the definition of the Fourier transform) and (here and elsewhere) $\xi \in \mathbb{R}$ represents the (Fourier) frequency coordinate. The choice of $[-\pi, \pi]$ in the following definition is thoroughly justified in annex B page 34 .

\section{Definition (The observable scene $u$.)}

We call observable scene any non negative deterministic function $u$ of the form $u=\mathbb{1}_{\left[-\frac{1}{2}, \frac{1}{2}\right]} * g * s$. Recall that the $\mathbb{1}_{\left[-\frac{1}{2}, \frac{1}{2}\right]}$ denotes the characteristic function of the interval $\left[-\frac{1}{2}, \frac{1}{2}\right]$ and is related to the normalized pixel sensor, the PSF is $g \in S(\mathbb{R})$ and $[-\pi, \pi]$ band limited and $s \in L_{l o c}^{1}(\mathbb{R})$ represents the (non negative) photon emission intensity. We have that $u \in L_{l o c}^{1}(\mathbb{R})$ and assume that $u$ satisfies $\mu:=\lim _{T R \rightarrow+\infty} \frac{1}{2 R} \int_{-R}^{R} u(x) d x \in \mathbb{R}^{+}$. In addition, we assume that $\tilde{u}:=u-\mu \epsilon$ $L^{1}(\mathbb{R}) \cap L^{2}(\mathbb{R})$.

Note that $u$ is the sum of the constant $\mu$ and of $\tilde{u} \in L^{1}(\mathbb{R})$. Thus, we have $u \in S^{\prime}(\mathbb{R})$ (the space of tempered distribution). This means that $u$ enjoys a Fourier transform in $S^{\prime}(\mathbb{R})$, see, e.g., [6, p. 173], see also [45, p. 23]. In addition, $u$ and $\tilde{u}$ inherit the frequency cut off of the PSF $g$. Therefore, $u$ and $\tilde{u}$ are $[-\pi, \pi]$ band limited. In addition note that the assumption $\tilde{u} \in L^{2}(\mathbb{R})$ is w.l.o.g. Indeed, since $\tilde{u} \in L^{1}(\mathbb{R})$, from Riemann-Lebesgue theorem (see e.g., [23, prop. 2.1]), we have that $\hat{\tilde{u}}$ is continuous. In addition, since $\tilde{u}$ is $[-\pi, \pi]$ is band limited we have that $\hat{\tilde{u}}$ is continuous and has compact support. We deduce that $\hat{\tilde{u}} \in L^{2}(\mathbb{R})$. Therefore, we obtain that $\tilde{u} \in L^{2}(\mathbb{R})$ w.l.o.g.

We can now give a definition of the observed sample at a pixel centered at $x$ that we shall denote $o b s(x)$.

Definition (Observed sample of a of pixel that includes any additive (sensor readout) noise of finite variance in addition to the Poisson photon (shot) noise.)

We assume that the observed sample produced by a unit pixel sensor centered at $x \in \mathbb{R}$ is corrupted by an additive noise $\eta(x)$ that we shall call readout noise. We assume that $\mathbb{E}(\eta(x))=0$ and that $\operatorname{var}(\eta(x))=\sigma_{r}<+\infty$. We shall denote this observed sample by obs(x), (from (9)), we have

$$
o b s(x) \sim \mathcal{P}\left(\int_{t_{1}}^{t_{2}} u(x-v t) d t\right)+\eta(x)
$$

where $\left[t_{1}, t_{2}\right]$ is the time exposure interval, the observable scene $u$ is defined by definition 2.1 page 8 and moves at velocity $v \in \mathbb{R}$. The notation $X \sim Y$ means that the random variables $X$ and $Y$ have the same law.

In the sequel we will need to compute MSEs as well as SNRs. Therefore, we will need to compute expected values and variances of the observed samples. Thus, we need to justify the validity of these operations. This is done in annex $\mathrm{C}$ page 35 . 
The definition 2.1 entails that $o b s(x)$, the observed sample of a pixel sensor centered at position $x$, is a measurable function (a random variable see, e.g., [44, p. 168]) for which it is mathematically possible to compute, e.g., the expectation and the variance.

The images produced by a digital camera are discrete. In addition, the image obtained by a coded exposure camera requires to undergo a deconvolution to get the final crisp image. Note that the Wiener filter (see, e.g., [22, p. 205], [9, p. 95], [55, p. 159] see also [10, p. 252] for a definition) is not applicable in our case since the Poisson noise is not white additive. The calculation of the adequate deconvolution filter requires a continuous model. Thus, we now turn to the sampling and interpolation in order to go comfortably from the discrete observations to the underlying continuous image.

\subsection{Sampling and interpolation}

This section recalls the principles of the Shannon-Whittaker interpolation that applies to, e.g., images that have the band limitedness structure. Consider a $[-\pi, \pi]$ band limited deterministic function $f \in L^{1}(\mathbb{R}) \cap L^{2}(\mathbb{R})$. From the values $f(n)$ for $n \in \mathbb{Z}$ the Shannon-Whittaker interpolation of $f$ is

$$
\sum_{n \in \mathbb{Z}} f(n) \operatorname{sinc}(x-n)\left(\text { where } \operatorname{sinc}(x)=\frac{\sin (\pi x)}{\pi x}\right)
$$

and the above series converges uniformly to $f(x)$ for any $x \in \mathbb{R}$ (see, e.g., [15, p. 354]).

We recall that annex $\mathrm{B}$ page 34 proves that it is no loss to assume that $u$ is $[-\pi, \pi]$ band limited. However, the sample obs $(x)$ defined in definition 2.1 page 8 produced by the sensor is noisy as it contains the Poisson photon (shot) noise and the additive sensor readout noise. This means that $o b s$ is not a deterministic function. The Shannon-Whittaker theorem is usually applied to deterministic functions. Some generalization exists in the case where the observed samples are corrupted by an additive noise, see, e.g., [26, p. 111], or to sample wide sense stationary stochastic signals, see, e.g., [26, p. 148]. However, the Poisson photon shot noise is not additive. Therefore, the first generalization is not applicable. In addition, from definition 2.1 page 8 we deduce that the autocorrelation function $\mathbb{E}(\operatorname{obs}(x) o b s(y))$ is not a function of the variable $x-y$. This means that the samples of a coded exposure camera cannot be seen as the samples of a wide sense stationary stochastic process (see, e.g., [9, p. 17] for the definition). Thus, to the best of our knowledge, the existing generalizations of the Shannon-Whittaker theorem are not sufficient to treat the observed samples of a coded exposure camera (defined in definition 2.1 page 8). Consequently, in the sequel, we shall carefully prove that, in fact, the Shannon-Whittaker theorem holds for the observed samples of a coded exposure camera. In other words, we shall prove that

$$
\text { "obs }(x)=\sum_{n \in \mathbb{Z}} o b s(n) \operatorname{sinc}(x-n) "\left(\text { where } \operatorname{sinc}(x)=\frac{\sin (\pi x)}{\pi x} .\right)
$$

is mathematically feasible for the obs defined in definition 2.1 page 8 .

Therefore, in the sequel, we assume that the observed samples are obtained from a sensor array and that the sensor array is designed according to the Shannon-Whittaker sampling theory. Thus, we assume that the samples obs $(x)$ are obtained at a unit rate, i.e., for $x \in \mathbb{Z}$. Consequently, we shall denote the observed samples by obs $(n)$. This means that, in the sequel, we shall neglect the boundaries effect due to the deconvolution. This is another way to get rid of the boundaries effects without assuming that the observed scene is periodic as required by linear algebra (with Toepliz matrices) model based (see, e.g., [2, 3, 4, 17, 38, 40, 47, 48]). 
(This is needed because, to the best of our knowledge, most natural scenes are not periodic.) Note that this is in favor of the coded exposure method since the support of the exposure function is larger than the support of the exposure function of a snapshot.

Hereinafter, we assume that the sequence of random variables $(\eta(n))_{n \in \mathbb{Z}}$ are mutually independent, identically distributed and independent from the shot noise, i.e., independent from $\mathcal{P}\left(\int_{t_{1}}^{t_{2}} u(n-v t) d t\right)$ where $n \in \mathbb{Z}$. This independence assumption represents no limitation for the model. Indeed, a photon is not caught twice by the sensor array. In addition, the additive (sensor readout) noise comes from an inaccurate reading of the sample value that does not depends on the Poisson photon (shot) noise.

We have defined the observed samples produced by a light sensor in definition 2.1 page 8 . This definition includes both the Poisson photon (shot) noise and an additive (readout) noise of finite variance. We now turn to the mathematical formalization of the coded exposure method.

\section{A mathematical model of coded exposure camera that in- cludes any additive (sensor readout) noise of finite variance in addition to the Poisson photon (shot) noise}

The goal of this section is to formalize the coded exposure method. In this section, we consider invertible and deterministic "exposure codes" and provides the MSE and SNR of these exposure strategies. The study yields to theorem 3.8 page 23 .

The coded exposure (flutter shutter) method permits to modulate, with respect to the time, the photons flux caught by the sensor array. Indeed, the Agrawal, Raskar et al. flutter shutter method $[2,3,4,37,38,39]$ consists in opening/closing the camera shutter on sub-intervals of the exposure time. In such a situation the exposure function that controls when the shutter is open or closed is binary and piecewise constant. Since it is piecewise constant it is possible to encode this function using an "exposure code". (We give a mathematical definition of these objects page 11.)

Note that neither the model nor the results of [48] can be used in this paper. Indeed, in [48] the additive (sensor readout) noise is neglected. Therefore, the formalism of [48] does not hold with the more elaborated set up that we shall consider here. Indeed, this paper considers any additive sensor readout noise of finite variance in addition to the Poisson photon (shot) noise.

As we have seen, in their seminal work [2, 3, 4, 37, 38, 39], Agrawal, Raskar et al. propose to use binary exposure codes. Yet, mathematically, we could envisage smoother exposure codes that are not binary. Indeed, with a bigger searching space for the exposure code the MSE and SNR can be expected to be better than with the smaller set of binary codes. Therefore, in the sequel, we shall assume that the exposure codes have values in $[0,1]$. The value 0 means that the shutter is closed while the value 1 means the shutter is open and, e.g., $\frac{1}{2}$ means that half of the photons are allowed to reach the sensor. We do not consider the practical feasibility of these non binary exposure codes as this is out of the scope of this paper which proposes a mathematical framework and formulae.

We first formalize the fact that the exposure code method modulates temporally the flux of photons that are allowed to reach the sensor by giving an adequate definition of a "exposure function" that, hereinafter, we shall denote $\alpha$. To be precise, the gain $\alpha(t)$ at time $t$ is defined as the proportion of photons that are allowed to travel to the sensor. We then give the formula of the observed samples taking the exposure function into account (see definition 3 page 11). 
Definition (Exposure function, exposure code.)

We call exposure function any function $\alpha$ of the form

$$
\begin{aligned}
\alpha: \mathbb{R} & \longrightarrow[0,1] \\
t & \longmapsto \sum_{k=-\infty}^{+\infty} \alpha_{k} \mathbb{1}_{[k \Delta t,(k+1) \Delta t)}(t) .
\end{aligned}
$$

We assume that $a_{k} \in[0,1]$, for any $k$, that $\left(a_{k}\right)_{k} \in \ell^{1}(\mathbb{Z})$ and that $\Delta t>0$. The sequence $\left(a_{k}\right)_{k}$ is called exposure code.

Remark $\left(\alpha \in L^{1}(\mathbb{R}) \cap L^{2}(\mathbb{R}) \cap L^{\infty}(\mathbb{R})\right)$

The above definition can cope with finitely supported codes, e.g., the Agrawal Raskar et al. code $\left[38\right.$, p. 5] and patent application $[39]$. Since, $\ell^{1}(\mathbb{Z}) \subset \ell^{2}(\mathbb{Z}) \subset \ell^{\infty}(\mathbb{Z})$, we deduce that for any exposure code we have $\alpha \in L^{1}(\mathbb{R}) \cap L^{2}(\mathbb{R}) \cap L^{\infty}(\mathbb{R})$.

We have defined the exposure function that controls with respect to the time the camera shutter. Recall that $\alpha(t)$ is nothing but the percentage of photons allowed to reach the sensor at time $t$. We are now in position to give the formula of the observed samples of a coded exposure camera.

Let $\alpha$ be an exposure function and $u(x-v t)$ a scene moving at a velocity $v \in \mathbb{R}$. The exposure function modulates with time the incoming photon flux. Therefore, from definition 2.1 page 8 , we deduce that $\operatorname{obs}(n)$, the observed sample at a position $n \in \mathbb{Z}$, is a random variable

$$
o b s(n) \sim \mathcal{P}\left(\int_{-\infty}^{\infty} \alpha(t) u(n-v t) d t\right)+\eta(n) \sim \mathcal{P}\left(\left(\frac{1}{|v|} \alpha\left(\frac{\dot{v}}{v}\right) * u\right)(n)\right)+\eta(n) .
$$

Recall that the random variables obs $(n)$ are only obtained for $n \in \mathbb{Z}$.

\section{Definition (Observed samples of a coded exposure camera.)}

Let $\alpha$ be an exposure function. We call observed samples at position $n$ of the scene $u$ (defined in definition 2.1 page 8 ) moving at velocity $v \in \mathbb{R}$ the random variable

$$
o b s(n) \sim \mathcal{P}\left(\left(\frac{1}{|v|} \alpha\left(\frac{\dot{v}}{v}\right) * u\right)(n)\right)+\eta(n) .
$$

Recall that the random variables $o b s(n) n \in \mathbb{Z}$ are mutually independent (see page 10). From definition 2.1 page 8 we have that $u$ is of the form $L^{1}(\mathbb{R})$ plus constant and is band limited. From definition 3 page 11 we have that $\alpha \in L^{1}(\mathbb{R})$. We obtain that the convolution in (11) is well defined everywhere. In addition, note that the pixels are read only once as in, e.g., $[2,3,4$, $37,38,39]$. This means that with a coded exposure camera only one image is observed, stored and transmitted.

Remark (The motion blur of a standard camera is not invertible as soon as its support exceeds two pixels.)

A standard camera can be seen as a coded exposure camera if the exposure function $\alpha$ is of the form $\alpha=\mathbb{1}_{[0, \Delta t]}$, where $\Delta t>0$ is the exposure time measured in second(s). Consider the idealistic noiseless case where, from (11), one would observe

$$
\mathbb{E}(o b s(n))=\left(\frac{1}{|v|} \mathbb{1}_{[0, \Delta t]}\left(\frac{\dot{v}}{v}\right) * u\right)(n) .
$$


From definition 2.1 page $8 u$ is $[-\pi, \pi]$ band limited. Therefore, we deduce that the convolution in (12) is non invertible as soon as the Fourier transform of $\frac{1}{|v|} \mathbb{1}_{[0, \Delta t]}(\dot{\dot{v}})$ vanishes on $[-\pi, \pi]$. For any $\xi \in \mathbb{R}$, we have $\mathcal{F}\left(\frac{1}{|v|} \mathbb{1}_{[0, \Delta t]}(\dot{\dot{v}})\right)(\xi)=\mathcal{F}\left(\mathbb{1}_{[0, \Delta t]}\right)(\xi v)$. In addition, from the definition of the Fourier transform (xv), for any $\xi \in \mathbb{R}$ we have

$$
\mathcal{F}\left(\mathbb{1}_{[0, \Delta t]}\right)(\xi)=\Delta t \operatorname{sinc}\left(\frac{\xi \Delta t}{2 \pi}\right) e^{\frac{-i \xi \Delta t}{2}} .
$$

Therefore, for any $\xi \in \mathbb{R}$, we have

$$
\mathcal{F}\left(\frac{1}{|v|} \mathbb{1}_{[0, \Delta t]}\left(\frac{\dot{v}}{v}\right)\right)(\xi)=\Delta t \operatorname{sinc}\left(\frac{\xi v \Delta t}{2 \pi}\right) e^{\frac{-i \xi v \Delta t}{2}} .
$$

From the definition (xvii) of the sinc function, we deduce that the convolution in (12) is not invertible as soon as the blur support $|v| \Delta t \geqslant 2$. Since, the velocity $v$ is measured in pixed per second, and the exposure time $\Delta t$ is measured in second(s) we deduce that as soon as the blur support $|v| \Delta t$ exceeds two pixels the motion blur of a standard camera is not invertible.

The observed samples of any coded exposure camera are formalized in definition 3 page 11 . We wish to compute the MSE and SNR of a deconvolved crisp image with respect to the continuous observable scene $u$ defined in definition 2.1 page 8 . The study yields to theorem 3.8 page 23 .

To compute the MSE and SNR, a continuous deconvolved crisp signal $\mathfrak{n}_{e s t}$ must be defined from the observed samples obs(n) observed for $n \in \mathbb{Z}$. Thus, our work-plan is

\section{Work-plan:}

Step 1 Justify the mathematical feasibility of the Shannon-Whittaker interpolation "obs $(x)=$ $\sum_{n \in \mathbb{Z}}$ obs $(n) \operatorname{sinc}(x-n)$ " of the observed samples obs $(n)$ (see definition 3 page 11) of a coded exposure camera. This is done in section 3.1. The study yields to proposition 3.4 page 16 .

Step 2 Deduce the conditions on the exposure function $\alpha$ (see definition 3 page 11) for the existence of an inverse filter $\gamma$ that deconvolve the observed image and gives back a crisp image. This is done in section 3.2. The study yields to proposition 3.7 page 19 .

Step 3 Compute the MSE and SNR of this deconvolved crisp image with respect to the continuous observable scene $u$ (see definition 2.1 page 8 ). This is treated in section 3.3. The study yields to theorem 3.8 page 23 .

In order to tackle the step 1 of our work plan, the following proposition and corollary will be needed to compute the MSE and SNR of the coded exposure method.

Proposition 3.1. The observed samples of the coded exposure method are such that, for any $n \in \mathbb{Z}$

$$
\mathbb{E}(o b s(n))=\left(\frac{1}{|v|} \alpha\left(\frac{\dot{v}}{v}\right) * u\right)(n) \text { and } \operatorname{var}(o b s(n))=\left(\frac{1}{|v|} \alpha\left(\frac{\dot{v}}{v}\right) * u\right)(n)+\sigma_{r}^{2} .
$$

Proof. The proof is a direct consequence of definition 3 page 11. 
Lemma 3.2. The deterministic function $\mathbb{R} \ni x \mapsto\left(\frac{1}{|v|} \alpha(\dot{v}) * u\right)(x)$ is $[-\pi, \pi]$ band limited, uniformly bounded and for any $x \in \mathbb{R}$ we have

$$
\left(\frac{1}{|v|} \alpha\left(\frac{1}{v}\right) * u\right)(x)=\left(\frac{1}{|v|} \alpha\left(\frac{\dot{v}}{v}\right) * \tilde{u}\right)(x)+\mu \int_{\mathbb{R}} \alpha(t) d t .
$$

Proof. See annex D page 35 .

Corollary 3.3. The deterministic function $\mathbb{Z} \ni n \mapsto \operatorname{var}(o b s(n))$ is uniformly bounded.

Proof. The proof is immediate from proposition 3.1 and lemma 3.2 .

We now turn to the step 1 of our work-plan that proves the mathematical feasibility of the Shannon-Whittaker interpolation, formally given in equation (10) page 9, of the sequence obs $(n)$ where $n \in \mathbb{Z}$ to a stochastic process obs $(x)$ where $x \in \mathbb{R}$.

\subsection{Mathematical feasibility of the Shannon-Whittaker interpolated image obs $(x)$}

This section tackles the step 1 of our work-plan. The study yields to proposition 3.4 page 16 . We give a rigorous mathematical proof of the mathematical feasibility of the Shannon-Whittaker interpolation of the sample obs $(n)$ obtained for $n \in \mathbb{Z}$. In other words, we need to find a suitable convergence, for any $x \in \mathbb{R}$, of the series formally defined by

$$
o b s(x)=\sum_{n=-\infty}^{\infty} o b s(n) \operatorname{sinc}(x-n) .
$$

Recall that we wish to compute the MSE and SNR of the final crisp (deconvolved) image. Therefore, we need closed formulae for the expectation and variance of (16). Thus, we need to prove that the series (16) converges, for any $x \in \mathbb{R}$, in quadratic mean. We shall use a Cauchy argument (see, e.g., [41, thm 6.6.2 p. 194]). We now give the construction details.

Consider the

$$
o \tilde{b} s(n):=o b s(n)-\mathbb{E}(o b s(n)),
$$

for $n \in \mathbb{Z}$. The proof is in three steps. The step a) proves that, for any $x \in \mathbb{R}$, the series

$$
\sum_{n=-\infty}^{+\infty} o \tilde{b} s(n) \operatorname{sinc}(x-n)
$$

converges in quadratic mean. The step $\mathrm{b}$ ) proves that, for any $x \in \mathbb{R}$, the series

$$
\sum_{n=-\infty}^{+\infty} \mathbb{E}(o b s(n)) \operatorname{sinc}(x-n)
$$

converges to a deterministic constant. These two steps entail that the series in (16) converges, for any $x \in \mathbb{R}$, in quadratic mean. The step c) provides explicit formulae for the expectation and variance of (16). Recall that these calculations will be needed for the computation of MSE and SNR of the deconvolved crisp image. We now turn to the proof of step a). 
Step a): From (17), for any $n \in \mathbb{Z}$ we have

$$
\mathbb{E}(o \tilde{b} s(n))=0 ; \operatorname{var}(o \tilde{b} s(n))=\mathbb{E}\left(o \tilde{b} s(n)^{2}\right)=\operatorname{var}(o b s(n)) .
$$

Consider the finite sums of independent random variables

$$
\tilde{o}^{N}(x):=\sum_{-N}^{N} o \tilde{b} s(n) \operatorname{sinc}(x-n) .
$$

Note that hereinafter, $\tilde{o}^{N}$ denotes the $N$-th term of the sequence defined in (19). For any $N \geqslant M$ and any $x \in \mathbb{R}$ we have that

$$
\mathbb{E}\left(\left|\tilde{o}^{N}(x)-\tilde{o}^{M}(x)\right|^{2}\right)=\sum_{M<|n| \leqslant N} \mathbb{E}\left((\tilde{o b} s(n))^{2}\right) \operatorname{sinc}^{2}(x-n) .
$$

Therefore, combining (18) and (20) we deduce that

$$
\mathbb{E}\left(\left|\tilde{o}^{N}(x)-\tilde{o}^{M}(x)\right|^{2}\right)=\sum_{M<|n| \leqslant N} \operatorname{var}(o b s(n)) \operatorname{sinc}^{2}(x-n),
$$

for any $x \in \mathbb{R}$. From corollary 3.3 page 13 we have that $\sup _{n} \operatorname{var}($ obs $(n))<+\infty$. Hence, from $(21)$ we have

$$
\mathbb{E}\left(\left|\tilde{o}^{N}(x)-\tilde{o}^{M}(x)\right|^{2}\right) \leqslant\left(\sup _{n} \operatorname{var}(\operatorname{obs}(n))\right) \sum_{M<|n| \leqslant N} \operatorname{sinc}^{2}(x-n),
$$

for any $x \in \mathbb{R}$. The series $\sum_{n=-\infty}^{+\infty} \operatorname{sinc}^{2}(x-n)$ converges for any $x \in \mathbb{R}$. Consequently, from (22), for any $x \in \mathbb{R}$ and $\epsilon>0$ we deduce that there exists $M_{0} \in \mathbb{N}$ such that if $M_{0} \leqslant M \leqslant N$ we have

$$
\mathbb{E}\left(\left|\tilde{o}^{N}(x)-\tilde{o}^{M}(x)\right|^{2}\right)<\epsilon .
$$

Therefore, from the Cauchy criterion (see, e.g., [41, thm 6.6.2 p. 194]) we deduce that, for any $x \in \mathbb{R}$, the series

$$
o \tilde{b} s(x)=\sum_{n=-\infty}^{+\infty} o \tilde{b} s(n) \operatorname{sinc}(x-n)
$$

converges in quadratic mean to a limit that we can therefore call or $s(x)$. This concludes step a). We now turn to step b) that proves that, for any $x \in \mathbb{R}$, the series $\sum_{n=-\infty}^{+\infty} \mathbb{E}($ obs $(n)) \operatorname{sinc}(x-n)$ converges to a deterministic constant. Recall that is needed to conclude that (16) page 13 converges in quadratic mean.

Step b): From proposition 3.1 page 12 , for any $x \in \mathbb{R}$, we have

$$
\sum_{n=-N}^{N} \mathbb{E}(\operatorname{obs}(n)) \operatorname{sinc}(x-n)=\sum_{n=-N}^{N}\left(\frac{1}{|v|} \alpha\left(\frac{\dot{v}}{v}\right) * u\right)(n) \operatorname{sinc}(x-n) .
$$

Combining (15) page 13 and (24), we have, for any $x \in \mathbb{R}$,

$$
\sum_{n=-N}^{N} \mathbb{E}(\operatorname{obs}(n)) \operatorname{sinc}(x-n)=\sum_{n=-N}^{N}\left(\frac{1}{|v|} \alpha\left(\frac{\dot{v}}{v}\right) * \tilde{u}\right)(n) \operatorname{sinc}(x-n)+\mu\left[\int_{\mathbb{R}} \alpha(t) d t\right] \sum_{n=-N}^{N} \operatorname{sinc}(x-n) .
$$


We first take care of the first term on the right hand side (RHS) of (25). From definition 2.1 page 8 we have $\tilde{u} \in L^{1}(\mathbb{R})$ and $\tilde{u}$ is $[-\pi, \pi]$ band limited. From definition 3 page $11 \alpha \in L^{1}(\mathbb{R})$. Therefore, from Young inequality (see, e.g., $\left[25\right.$, p. 525]) we have that $\left(\frac{1}{|v|} \alpha(\dot{\bar{v}}) * \tilde{u}\right) \in L^{1}(\mathbb{R})$ and is $[-\pi, \pi]$ band limited since $\tilde{u}$ is band limited in this range. Hence, we deduce (see, e.g., $[15$, p. 354]) that, for any $x \in \mathbb{R}$,

$$
\sum_{n=-\infty}^{+\infty}\left(\frac{1}{|v|} \alpha\left(\frac{\dot{v}}{v}\right) * \tilde{u}\right)(n) \operatorname{sinc}(x-n)=\left(\frac{1}{|v|} \alpha\left(\frac{\dot{v}}{v}\right) * \tilde{u}\right)(x) .
$$

We now take care of the second term on the RHS of (25). For any $x \in \mathbb{R}$, we have $\sum_{n=-\infty}^{+\infty} \operatorname{sinc}(x-$ $n)=1$. Thus, combining (25) and (26) we have that, for any $x \in \mathbb{R}$,

$$
\sum_{n=-\infty}^{+\infty} \mathbb{E}(o b s(n)) \operatorname{sinc}(x-n)=\left(\frac{1}{|v|} \alpha\left(\frac{\dot{v}}{v}\right) * \tilde{u}\right)(x)+\mu\left[\int_{\mathbb{R}} \alpha(t) d t\right] .
$$

From definition 2.1 page 8 , for any $x \in \mathbb{R}$ we have $u(x)=\tilde{u}(x)+\mu$. In addition, $\int_{-\infty}^{\infty} \frac{1}{|v|} \alpha\left(\frac{t}{v}\right) \mu d t=$ $\mu \int_{-\infty}^{\infty} \alpha(t) d t$. Hence, from (27) we obtain that, for any $x \in \mathbb{R}$,

$$
\sum_{n=-\infty}^{+\infty} \mathbb{E}(o b s(n)) \operatorname{sinc}(x-n)=\left(\frac{1}{|v|} \alpha\left(\frac{\dot{v}}{v}\right) * u\right)(x) .
$$

This concludes step b).

We now combine the steps a) and b). Combining (16) and (17) page 13, the series

$$
\sum_{n=-\infty}^{+\infty} o b s(n) \operatorname{sinc}(x-n)=\sum_{n=-\infty}^{+\infty} o \tilde{b} s(n) \operatorname{sinc}(x-n)+\sum_{n=-\infty}^{+\infty} \mathbb{E}(o b s(n)) \operatorname{sinc}(x-n)
$$

converges, for any $x \in \mathbb{R}$, in quadratic mean to a limit that we can therefore call obs $(x)$. Indeed, from step a), it is the sum of the quadratic mean convergent series $\sum_{n=-N}^{N} o \tilde{b} s(n) \operatorname{sinc}(x-n)$ and, from (28), of the deterministic constant $\left(\frac{1}{|v|} \alpha(\dot{\bar{v}}) * u\right)(x)$. Consequently, we deduce that, for any $x \in \mathbb{R}$, the series defined in (16) page 13 converges in quadratic mean. Thus, we call obs $(x)$, for any $x \in \mathbb{R}$, this limit. We now turn to step c) that gives explicit formulae for the expectation and variance of the quadratic mean convergent series obs $(x)$ defined in (16).

Step c): The convergence in quadratic mean implies the convergence of the two firsts moments (see [20, Ex 5.6 (a)-(b), p. 158]). Therefore, for any $x \in \mathbb{R}$ we have

$$
\mathbb{E}\left(\sum_{n=-N}^{N} \text { obs }(n) \operatorname{sinc}(x-n)\right) \stackrel{N \rightarrow \infty}{\longrightarrow} \mathbb{E}\left(\sum_{n=-\infty}^{\infty} o b s(n) \operatorname{sinc}(x-n)\right)
$$

and

$$
\operatorname{var}\left(\sum_{n=-N}^{N} \operatorname{obs}(n) \operatorname{sinc}(x-n)\right) \stackrel{N \rightarrow \infty}{\longrightarrow} \operatorname{var}\left(\sum_{n=-\infty}^{\infty} o b s(n) \operatorname{sinc}(x-n)\right) .
$$

In addition, the linearity of the expectation and the independence of the observed samples $o b s(n)$ imply that, for any $x \in \mathbb{R}$,

$$
\mathbb{E}\left(\sum_{n=-N}^{N} \operatorname{obs}(n) \operatorname{sinc}(x-n)\right)=\sum_{n=-N}^{N} \mathbb{E}(\text { obs }(n)) \operatorname{sinc}(x-n)
$$


and

$$
\operatorname{var}\left(\sum_{n=-N}^{N} \operatorname{obs}(n) \operatorname{sinc}(x-n)\right)=\sum_{n=-N}^{N} \operatorname{var}(o b s(n)) \operatorname{sinc}^{2}(x-n) .
$$

Therefore, combining (29) with (31) and the definition (16) page 13 of $o b s(x)$ we have

$$
\sum_{n=-N}^{N} \mathbb{E}(o b s(n)) \operatorname{sinc}(x-n) \stackrel{N \rightarrow \infty}{\longrightarrow} \mathbb{E}(o b s(x)),
$$

for any $x \in \mathbb{R}$. Similarly, combining (30) with (32) and the definition (16) page 13 of obs(x) we have that

$$
\sum_{n=-N}^{N} \operatorname{var}(\text { obs }(n)) \operatorname{sinc}(x-n) \stackrel{N \rightarrow \infty}{\longrightarrow} \operatorname{var}(\text { obs }(x)),
$$

for any $x \in \mathbb{R}$. Combining (28) and (33) we have that, for any $x \in \mathbb{R}$,

$$
\mathbb{E}(o b s(x))=\left(\frac{1}{|v|} \alpha\left(\frac{\dot{v}}{v}\right) * u\right)(x) .
$$

From proposition 3.1 page 12 and (34) we deduce that, for any $x \in \mathbb{R}$,

$$
\sum_{n=-N}^{N}\left[\left(\frac{1}{|v|} \alpha\left(\frac{\dot{v}}{v}\right) * u\right)(n)+\sigma_{r}^{2}\right] \operatorname{sinc}^{2}(x-n) \stackrel{N \rightarrow \infty}{\longrightarrow} \operatorname{var}(o b s(x)) .
$$

Since, for any $x \in \mathbb{R}$, we have $\sum_{n=-\infty}^{+\infty} \operatorname{sinc}^{2}(x-n)=1$, from (36) we deduce that, for any $x \in \mathbb{R}$,

$$
\operatorname{var}(o b s(x))=\sum_{n=-\infty}^{+\infty}\left[\left(\frac{1}{|v|} \alpha\left(\frac{\dot{v}}{v}\right) * u\right)(n) \operatorname{sinc}^{2}(x-n)\right]+\sigma_{r}^{2} .
$$

In addition, from corollary 3.3 page 13 , we deduce that, for any $x \in \mathbb{R}, \operatorname{var}(o b s(x))$ is finite.

Thus, we proved

Proposition 3.4. (Mathematical feasibility of the Shannon-Whittaker interpolation of the observed samples obs $(n) n \in \mathbb{Z}$.)

The Shannon-Whittaker interpolation of the observed samples obs $(n) n \in \mathbb{Z}$ defined in definition 3 page 11 is, for any $x \in \mathbb{R}$, the quadratic mean convergent series

$$
o b s(x)=\sum_{n=-\infty}^{+\infty} o b s(n) \operatorname{sinc}(x-n) .
$$

From (35), its expectation is, for any $x \in \mathbb{R}$,

$$
\mathbb{E}(o b s(x))=\left(\frac{1}{|v|} \alpha\left(\frac{\dot{v}}{v}\right) * u\right)(x) .
$$

From (37), its variance is finite and, for any $x \in \mathbb{R}$,

$$
\operatorname{var}(o b s(x))=\sum_{n=-\infty}^{+\infty}\left[\left(\frac{1}{|v|} \alpha\left(\frac{\dot{v}}{v}\right) * u\right)(n) \operatorname{sinc}^{2}(x-n)\right]+\sigma_{r}^{2} .
$$

We proved that the Shannon-Whittaker interpolation is mathematically feasible for the observed samples of a coded exposure camera defined in definition 3 page 11. We shall now turn to the definition of the deconvolved crisp image that we shall denote $\mathbb{d}_{e s t}(x)$. 


\subsection{Mathematical feasibility of the the deconvolved crisp image $\mathbb{d}_{e s t}(x)$}

This section treats the step 2 of our work-plan. The study yields to proposition 3.7 page 19 . Recall that the existence of $o b s(x)=\sum_{n=-\infty}^{+\infty}$ obs $(n) \operatorname{sinc}(x-n)$ is given in proposition 3.4 page 16 .

Formally, we wish to consider $o b s * \gamma$ where $\gamma$ will be the deconvolution filter that inverses the convolution by the exposure function $\frac{1}{|v|} \alpha(\dot{\bar{v}})$ in proposition 3.4 equation (38). Note that we cannot recur to a Wiener filter to define $\gamma$. Indeed, due to the Poisson photon (shot) noise, the noise in of our observations obs $(n)$ defined in definition 3 page 11 is not white additive. Therefore, to the best of our knowledge, a Wiener filter is not defined (see, e.g., [22, p. 205], [9, p. 95], [55, p. 159] see also [10, p. 252] for a definition). Instead of using a Wiener filter we shall propose a filter designed so that the restored crisp image $\mathbb{d}_{e s t}$ is unbiased.

Beside the definition of the inverse filter $\gamma$, we need to prove the mathematical feasibility of "obs * $\gamma$ ". This is needed because obs given in proposition 3.4 page 16 does not belong to a classic Lebesgue space. In addition we need to deduce the conditions on the exposure function $\alpha$ (see definition 3 page 11) to guarantee that $\mathbb{E}(\gamma * o b s(x))=u(x)$ for any $x \in \mathbb{R}$, i.e., the conditions on $\alpha$ so that its convolution can be inverted.

We assume that the exposure function $\alpha \in L^{1}(\mathbb{R}) \cap L^{2}(\mathbb{R}) \cap L^{\infty}(\mathbb{R})$ defined in definition 3 page 11 satisfies $\hat{\alpha}(\xi v) \neq 0$ for any $\xi \in[-\pi, \pi]$. Under that condition the convolution $\left(\frac{1}{|v|} \alpha(\dot{v})\right) *$ $u$ is invertible because $u$ is $[-\pi, \pi]$ band-limited. Thus, provided $\hat{\alpha}(\xi v) \neq 0$ for any $\xi \in[-\pi, \pi]$, we can consider the inverse filter (defined by its inverse Fourier transform (xv))

$$
\gamma(x):=\mathcal{F}^{-1}\left(\frac{\mathbb{1}_{[-\pi, \pi]}(\xi)}{\hat{\alpha}(\xi v)}\right)(x) .
$$

This filter $\gamma$ will be applied to the Shannon-Whittaker interpolated image obs $(x)$ with $x \in \mathbb{R}$ given in proposition 3.4 page 16 . Since we assume that $\hat{\alpha}(\xi v) \neq 0$ for any $\xi \in[-\pi, \pi]$ we have $|\hat{\alpha}(\xi v)|>0$ for any $\xi \in[-\pi, \pi]$. Hence, we deduce that $\mathbb{R} \ni \xi \mapsto \frac{\mathbb{1}_{[-\pi, \pi]}(\xi)}{\hat{\alpha}(\xi v)}$ is bounded, has compact support and therefore belongs to $L^{1}(\mathbb{R}) \cap L^{2}(\mathbb{R})$. From its definition (39) and Riemann-Lebesgue theorem (see e.g. [23, prop. 2.1]) we have that $\gamma$ is continuous. In addition, we have $\gamma \in L^{2}(\mathbb{R})$ as the inverse Fourier transform of a function that belongs to $L^{2}(\mathbb{R})$. Thus, we deduce that

$$
\gamma^{2}(x) \leqslant \frac{C}{1+x^{2}} \forall x \in \mathbb{R}
$$

for some constant $C>0$. Furthermore from (39) we have $\hat{\gamma}(\xi)=\frac{\mathbb{1}_{[-\pi, \pi]}(\xi)}{\hat{\alpha}(\xi v)}$. Therefore, we have

$$
\hat{\gamma}(0)=\frac{1}{\hat{\alpha}(0)}=\frac{1}{\int_{\mathbb{R}} \alpha(x) d x} .
$$

Before we turn to the mathematical feasibility of "obs* $\gamma$ ", we first prove that the inverse filter $\gamma$ defined in (39) gives an unbiased estimator of $u$ that is $\mathbb{E}(o b s) * \gamma=u$. Adhuc, we used the Poisson summation formula (see annex G page 39) to prove equalities like " $\sum_{n=-\infty}^{\infty} \gamma(x-n)=\hat{\gamma}(0)$ ". The use of the Poisson summation formula requires deterministic functions that belongs to $L^{1}(\mathbb{R})$. Since $\gamma \in L^{2}(\mathbb{R})$ but $\alpha \notin L^{1}(\mathbb{R})$ we cannot recur to the Poisson summation formula (ix) used elsewhere. Instead we introduce that following lemma

Lemma 3.5. (Poisson like formula for the inverse filter $\gamma_{.}$)

For any $x \in \mathbb{R}$, we have

$$
\sum_{n=-\infty}^{+\infty} \gamma(x-n)=\frac{1}{\int_{\mathbb{R}} \alpha(x) d x}
$$


for $\gamma$ defined by (39).

Proof. The proof is in annex E page 35.

The next lemma means that in the idealistic noiseless case where one observes $\mathbb{E}($ obs $(n))$ for $n \in \mathbb{Z}$, the inverse filter defined in (39) page 17 allows us to get back the ideal crisp image $u$. This lemma will be useful in the noisy (general) case and to compute the MSE and SNR of the coded exposure method.

Lemma 3.6. (The inverse filter $\gamma$ gives back an unbiased estimator of $u$.

Provided that $\hat{\alpha}(\xi v) \neq 0$ for any $\xi \in[-\pi, \pi]$, we have

$$
\sum_{n=-\infty}^{+\infty}\left(\frac{1}{|v|} \alpha\left(\frac{\dot{v}}{v}\right) * u\right)(n) \gamma(x-n)=u(x),
$$

for any $x \in \mathbb{R}$ and $\gamma$ defined by (39) page 17 .

Proof. The proof is in annex F page 36 .

The lemma 3.6 page 18 proves that, at least in the idealistic noiseless case, i.e., when one observes the noiseless samples $\mathbb{E}(o b s(n))$ for $n \in \mathbb{Z}$, the inverse filter $\gamma$ is able to recover a crisp image. However, in a practical situation the observed samples are noisy. Therefore, we now turn to the general noisy case.

In order to get an unbiased estimate of $u$, i.e., a final crisp image we would like to define

$$
" \mathrm{u}_{e s t}:=o b s * \gamma " .
$$

In the sequel, using lemma 3.6 page 18 , we shall prove that $\mathbb{E}\left(\mathbb{u}_{\text {est }}(x)\right)=u(x)$ for any $x \in \mathbb{R}$. (This means that $\mathbb{u}_{e s t}$ is an unbiased estimator of $u$.) However, the convolution in (44) is undefined because the random function obs that comes from the Shannon-Whittaker interpolation of the observed samples defined in proposition 3.4 page 16 does not belong to any Lebesgue space. Therefore, we now justify the mathematical feasibility of (44) that (formally) defines the final crisp image.

From its definition (39) page 17 , we have that $\hat{\gamma}$ is compactly supported (on $[-\pi, \pi]$ ) and bounded. Therefore, $\hat{\gamma} \in L^{2}(\mathbb{R}) \cap L^{1}(\mathbb{R})$. From $\hat{\gamma} \in L^{1}(\mathbb{R})$ and Riemann-Lebesgue theorem (see e.g. $\left[23\right.$, prop. 2.1]) we deduce that $\gamma$ is bounded. In addition, from $\hat{\gamma} \in L^{2}(\mathbb{R})$ we have that $\gamma \in L^{2}(\mathbb{R})$. Consequently, $\gamma$ is $[-\pi, \pi]$ band-limited and $C^{\infty}(\mathbb{R})$, bounded, and belongs to $L^{2}(\mathbb{R})$. This means that $\gamma$ is a nice inverse filter.

We need to recur, again, to a convergence argument for the definiteness of $\mathbb{w}_{e s t}$. Since, $\gamma$ is $[-\pi, \pi]$ band limited, for any $x \in \mathbb{R}$, we have $(\operatorname{sinc}(\cdot-n) * \gamma)(x)=\gamma(x-n)$. Therefore, for any $x \in \mathbb{R}$, we set

$$
\mathbb{u}_{e s t}^{N}(x):=\sum_{n=-N}^{N} \operatorname{obs}(n) \gamma(x-n) .
$$

Note that hereinafter, $\mathfrak{u}_{e s t}^{N}(x)$ denotes the $N$-th term of the sequence defined in (45).

Our goal is to find an adequate mode of convergence, for any $x \in \mathbb{R}$, of these finite sums to

$$
\mathbb{u}_{e s t}(x):=\sum_{n=-\infty}^{\infty} \operatorname{obs}(n) \gamma(x-n) .
$$


From (45), for any $x \in \mathbb{R}$, we have

$$
\mathbb{E}\left(\mathbb{u}_{e s t}^{N}(x)\right)=\sum_{n=-N}^{N} \mathbb{E}(o b s(n)) \gamma(x-n) .
$$

Combining proposition 3.1 page 12 and (46), for any $x \in \mathbb{R}$, we have

$$
\mathbb{E}\left(\mathbb{u}_{\text {est }}^{N}(x)\right)=\sum_{n=-N}^{N}\left[\left(\frac{1}{|v|} \alpha\left(\frac{\dot{v}}{v}\right) * u\right)(n)\right] \gamma(x-n) .
$$

From lemma 3.6 page 18 we deduce that, for any $x \in \mathbb{R}$,

$$
\mathbb{E}\left(\mathrm{u}_{\text {est }}^{N}(x)\right) \stackrel{N \rightarrow \infty}{\longrightarrow} u(x) .
$$

We have

\section{Proposition 3.7. (Validity/Existence of the crisp deconvolved image $\mathbb{a}_{e s t}$. )}

Consider the observed samples obs( $n)$ defined in definition 3 page 11 and the inverse filter $\gamma$ defined in (39) page 17. We have that, for any $x \in \mathbb{R}$, the series

$$
\mathbb{u}_{e s t}(x):=\sum_{n=-\infty}^{\infty} o b s(n) \gamma(x-n)
$$

converges in quadratic mean. Furthermore, for any $x \in \mathbb{R}$, its expectation is

$$
\mathbb{E}\left(\mathbb{v}_{e s t}(x)\right)=u(x)
$$

and its variance is finite and

$$
\operatorname{var}\left(\mathbb{u}_{e s t}(x)\right)=\sum_{n=-\infty}^{\infty} \operatorname{var}(o b s(n))(\gamma(x-n))^{2} .
$$

This proposition means that $\mathbb{u}_{e s t}$ is an unbiased estimator of the observable scene $u$.

Proof. The proof follows exactly the same arguments as for the construction of $o b s(x)$ leading to proposition 3.4 page 16. Indeed, the decay of $\gamma$ given in (40) page 17 is the same as the sinc. Therefore, we obtain that, for any $x \in \mathbb{R}, \mathbb{w}_{e s t}^{N}(x)=\sum_{n=-N}^{N} \operatorname{obs}(n) \operatorname{sinc}(x-n)$ converges, in quadratic mean, to a limit that we can therefore call $\mathbb{n}_{e s t}(x)$. This mode of convergence implies the convergence of the two firsts moments (see [20, Ex 5.6 (a)-(b), p. 158]). Therefore, we again obtain that, for any $x \in \mathbb{R}, \mathbb{E}\left(\mathbb{u}_{\text {est }}^{N}(x)\right) \stackrel{N \rightarrow+\infty}{\longrightarrow} \mathbb{E}\left(\mathbb{u}_{\text {est }}(x)\right)$. Thus, from (48) we deduce that, for any $x \in \mathbb{R}, \mathbb{E}\left(\mathfrak{u}_{e s t}(x)\right)=u(x)$. In addition, for any $x \in \mathbb{R}$, we have $\operatorname{var}\left(\mathbb{u}_{\text {est }}^{N}(x)\right) \stackrel{N \rightarrow+\infty}{\longrightarrow} \operatorname{var}\left(\mathbb{u}_{\text {est }}(x)\right)$. Furthermore, the independence of $o b s(n)$ implies that, for any $x \in \mathbb{R}, \operatorname{var}\left(\mathbb{u}_{e s t}^{N}(x)\right)=\sum_{-N}^{N} \operatorname{var}(o b s(n)) \gamma^{2}(x-n)$. Thus, we have $\operatorname{var}\left(\mathbb{u}_{\text {est }}(x)\right)=$ $\sum_{-\infty}^{+\infty} \operatorname{var}(\operatorname{obs}(n)) \gamma^{2}(x-n)<+\infty$ for any $x \in \mathbb{R}$. Indeed, from corollary 3.3 page 13 , we have $\sup _{n} \operatorname{var}($ obs $(n))<+\infty$. From its definition (39) page 17 we have that $\gamma$ is $[-\pi, \pi]$ band-limited. In addition, from (40) page 17 we have that $\gamma^{2} \in L^{1}(\mathbb{R})$. Hence, we deduce that $\gamma^{2}$ is such that $\widehat{\gamma^{2}}(\xi)$ is supported on $[-2 \pi, 2 \pi]$. Moreover, from the definition of $\gamma(39)$ page 17 we have

$$
\widehat{\gamma^{2}}(2 \pi)=(\hat{\gamma} * \hat{\gamma})(2 \pi)=\int_{-\pi}^{\pi} \frac{\mathbb{1}_{[-\pi, \pi]}(2 \pi-\xi)}{\hat{\alpha}(v \xi) \hat{\alpha}(v(2 \pi-\xi))} d \xi .
$$


The integrand in (49) is non zero only on the zero Lebesgue measure set $\{\pi\}$. Therefore, we have $\widehat{\gamma^{2}}(2 \pi)=0$. Similarly, we have $\widehat{\gamma^{2}}(-2 \pi)=0$. Thus, from the Poisson formula (see annex G page 39) we deduce that

$$
\sum_{n=-\infty}^{+\infty} \widehat{\gamma^{2}}(2 \pi n)=\widehat{\gamma^{2}}(0)=\sum_{n=-\infty}^{+\infty} \gamma^{2}(x-n)=\|\gamma\|_{L^{2}(\mathbb{R})}^{2}
$$

From corollary 3.3 page 13 we have $\sup _{n} \operatorname{var}($ obs $(n))<+\infty$. Hence, for any $x \in \mathbb{R}$ we have $\operatorname{var}\left(\mathbb{u}_{e s t}(x)\right) \leqslant \sup _{n} \operatorname{var}($ obs $(n))\|\gamma\|_{L^{2}(\mathbb{R})}<+\infty$ which concludes the proof.

Proposition 3.7 page 19 proves the feasibility of the restore crisp image $\mathfrak{d}_{e s t}$. This concludes the step 2 of our work plan. We now turn to the step 3 of our work plan that is the calculations of the MSE and SNR of $\mathfrak{n}_{e s t}$ with respect to the observable scene $u$ (see definition 2.1 page 8).

\subsection{The MSE and SNR of the restored crisp image $\mathbb{t}_{e s t}$}

We have defined the deconvolved crisp image $\mathbb{n}_{e s t}$ in proposition 3.7 page 19 . We shall now turn to the step 3 of our work-plan that provides closed formulae for the average MSE and SNR of the coded exposure method. The study yields to theorem 3.8 page 23 .

Recall that, from proposition 3.7 page 19 , for any $x \in \mathbb{R}$, we have

$$
\mathbb{E}\left(\mathfrak{u}_{e s t}(x)\right)=u(x) .
$$

Therefore, for any $x \in \mathbb{R}$, we have

$$
\mathbb{E}\left(\left|\mathbf{u}_{e s t}(x)-u(x)\right|^{2}\right)=\operatorname{var}\left(\mathfrak{u}_{e s t}(x)\right) .
$$

In order to provide closed formulae for the MSE and SNR we need to evaluate $\operatorname{var}\left(\mathbb{u}_{e s t}(x)\right)$ and the limit of $\frac{1}{2 R} \int_{-R}^{R} \operatorname{var}\left(\mathbb{u}_{e s t}(x)\right) d x$ when $R \rightarrow+\infty$.

We now calculate $\operatorname{var}\left(\mathbb{v}_{e s t}(x)\right)$. From proposition 3.7 page 19 , for any $x \in \mathbb{R}$, we have

$$
\operatorname{var}\left(\mathbb{u}_{e s t}(x)\right)=\sum_{n=-\infty}^{\infty} \operatorname{var}(o b s(n))(\gamma(x-n))^{2} .
$$

Combining (14) page 12 and (53), for any $x \in \mathbb{R}$, we have

$$
\operatorname{var}\left(\mathbb{u}_{e s t}(x)\right)=\sum_{n=-\infty}^{\infty}\left[\left(\left(\frac{1}{|v|} \alpha\left(\frac{\dot{v}}{v}\right) * u\right)(n)+\sigma_{r}^{2}\right)(\gamma(x-n))^{2}\right] .
$$

Therefore, from (54) we deduce that, for any $x \in \mathbb{R}$,

$$
\operatorname{var}\left(\mathbb{u}_{e s t}(x)\right)=\sum_{n=-\infty}^{\infty}\left[\left(\frac{1}{|v|} \alpha\left(\frac{\dot{v}}{v}\right) * u\right)(n)(\gamma(x-n))^{2}\right]+\sigma_{r}^{2} \sum_{n=-\infty}^{\infty}(\gamma(x-n))^{2} .
$$

From definition 3 page 11, we have that $\alpha$ is non negative. Therefore, combining (15) page 13 and (55), for any $x \in \mathbb{R}$, we have

$$
\operatorname{var}\left(\mathfrak{u}_{e s t}(x)\right)=\sum_{n=-\infty}^{\infty}\left[\left(\frac{1}{|v|} \alpha\left(\frac{\dot{v}}{v}\right) * \tilde{u}\right)(n)(\gamma(x-n))^{2}\right]+\left(\mu\|\alpha\|_{L^{1}(\mathbb{R})}+\sigma_{r}^{2}\right) \sum_{n=-\infty}^{\infty}(\gamma(x-n))^{2} .
$$


Hence, combining (50) page 20 and (56), for any $x \in \mathbb{R}$, we have

$$
\operatorname{var}\left(\mathbb{u}_{e s t}(x)\right)=\sum_{n=-\infty}^{\infty}\left[\left(\frac{1}{|v|} \alpha\left(\frac{\dot{v}}{v}\right) * \tilde{u}\right)(n)(\gamma(x-n))^{2}\right]+\left(\mu\|\alpha\|_{L^{1}(\mathbb{R})}+\sigma_{r}^{2}\right)\|\gamma\|_{L^{2}(\mathbb{R})}^{2} .
$$

Moreover, from the definition of $\gamma(39)$ page 17 and Plancherel identity (146) we have

$$
\|\gamma\|_{L^{2}(\mathbb{R})}^{2}=\frac{1}{2 \pi} \int_{-\infty}^{+\infty} \frac{1}{|\hat{\alpha}(\xi v)|^{2}} \mathbb{1}_{[-\pi, \pi]}(\xi) d \xi
$$

Therefore, $\|\alpha\|_{L^{1}(\mathbb{R})}=\int_{\mathbb{R}} \alpha(t) d t$. Thus, combining (57) and (58) we deduce that, for any $x \in \mathbb{R}$,

$$
\operatorname{var}\left(\mathfrak{u}_{e s t}(x)\right)=\sum_{n=-\infty}^{\infty}\left[\left(\frac{1}{|v|} \alpha\left(\frac{\dot{v}}{v}\right) * \tilde{u}\right)(n)(\gamma(x-n))^{2}\right]+\frac{\mu\|\alpha\|_{L^{1}(\mathbb{R})}+\sigma_{r}^{2}}{2 \pi} \int_{\mathbb{R}} \frac{1}{|\hat{\alpha}(\xi v)|^{2}} \mathbb{1}_{[-\pi, \pi]}(\xi) d \xi .
$$

Hence, combining (52) and (59), for any $x \in \mathbb{R}$, we have

$$
\mathbb{E}\left(\left|\mathbb{u}_{e s t}(x)-u(x)\right|^{2}\right)=\sum_{n=-\infty}^{\infty}\left[\left(\frac{1}{|v|} \alpha\left(\frac{\dot{v}}{v}\right) * \tilde{u}\right)(n)(\gamma(x-n))^{2}\right]+\frac{\mu\|\hat{\alpha}\|_{L^{1}(\mathbb{R})}+\sigma_{r}^{2}}{2 \pi} \int_{\mathbb{R}} \frac{\mathbb{1}_{[-\pi, \pi]}(\xi) d \xi}{|\hat{\alpha}(\xi v)|^{2}} .
$$

We now calculate the limit of $\frac{1}{2 R} \int_{-R}^{R} \operatorname{var}\left(\mathfrak{u}_{e s t}(x)\right) d x$ when $R \rightarrow+\infty$. The function $\mathbb{R} \ni x \mapsto$ $\sum_{n=-\infty}^{\infty}\left[\left(\frac{1}{|v|} \alpha^{2}(\dot{\bar{v}}) * \tilde{u}\right)(n)(\gamma(x-n))^{2}\right]$ belongs to $L^{1}(\mathbb{R})$. Indeed, we have

$$
\int_{-\infty}^{+\infty}\left|\sum_{n=-\infty}^{\infty}\left[\left(\frac{1}{|v|} \alpha\left(\frac{\dot{v}}{v}\right) * \tilde{u}\right)(n)(\gamma(x-n))^{2}\right]\right| d x \leqslant \int_{-\infty}^{+\infty} \sum_{n=-\infty}^{\infty}\left[\left|\left(\frac{1}{|v|} \alpha(\dot{\dot{v}}) * \tilde{u}\right)(n)\right|(\gamma(x-n))^{2}\right] d x .
$$

Hence, it follows from (61) and Fubini theorem (see, e.g., [44, p. 196]) that

$$
\int_{-\infty}^{+\infty}\left|\sum_{n=-\infty}^{\infty}\left[\left(\frac{1}{|v|} \alpha\left(\frac{\dot{v}}{v}\right) * \tilde{u}\right)(n)(\gamma(x-n))^{2}\right]\right| d x \leqslant \sum_{n=-\infty}^{\infty}\left[\left|\left(\frac{1}{|v|} \alpha\left(\frac{\dot{v}}{v}\right) * \tilde{u}\right)(n)\right| \int_{-\infty}^{+\infty}(\gamma(x-n))^{2} d x\right] .
$$

For any $n \in \mathbb{Z}$, we have

$$
\int_{-\infty}^{+\infty}(\gamma(x-n))^{2} d x=\|\gamma\|_{L^{2}(\mathbb{R})}^{2}
$$

Therefore, from (62) we deduce that

$$
\int_{-\infty}^{+\infty}\left|\sum_{n=-\infty}^{\infty}\left[\left(\frac{1}{|v|} \alpha\left(\frac{\dot{v}}{v}\right) * \tilde{u}\right)(n)(\gamma(x-n))^{2}\right]\right| d x \leqslant\|\gamma\|_{L^{2}(\mathbb{R})}^{2} \sum_{n=-\infty}^{\infty}\left|\left(\frac{1}{|v|} \alpha(\dot{v}) * \tilde{u}\right)(n)\right| .
$$

From definition 2.1 page 8 we have $\tilde{u} \in L^{1}(\mathbb{R})$ and $\tilde{u}$ is $[-\pi, \pi]$ band limited. From definition 3 page $11 \alpha \in L^{1}(\mathbb{R})$. Therefore, from the Poisson summation formula (see annex G page 39) we obtain that

$$
\sum_{n=-\infty}^{\infty}\left|\left(\frac{1}{|v|} \alpha\left(\frac{\dot{v}}{v}\right) * \tilde{u}\right)(n)\right|=\left\|\frac{1}{|v|} \alpha\left(\frac{\dot{v}}{v}\right) * \tilde{u}\right\|_{L^{1}(\mathbb{R})}<+\infty .
$$


Indeed, from Young inequality (see, e.g., [25, p. 525]) we have that $\left(\frac{1}{|v|} \alpha(\dot{\bar{v}}) * \tilde{u}\right) \in L^{1}(\mathbb{R})$. In addition, from (40) page $17 \gamma \in L^{2}(\mathbb{R})$. Thus, combining (63) and (64) we deduce that

$$
\int_{-\infty}^{+\infty}\left|\sum_{n=-\infty}^{\infty}\left[\left(\frac{1}{|v|} \alpha\left(\frac{\dot{v}}{v}\right) * \tilde{u}\right)(n)(\gamma(x-n))^{2}\right]\right| d x<+\infty .
$$

From (65), we deduce that

$$
\frac{1}{2 R} \int_{-R}^{R} \sum_{n=-\infty}^{\infty}\left[\left(\frac{1}{|v|} \alpha\left(\frac{\dot{v}}{v}\right) * \tilde{u}\right)(n)(\gamma(x-n))^{2}\right] d x \stackrel{R \rightarrow+\infty}{\longrightarrow} 0 .
$$

For any $R>0$, from (59) we have that

$$
\begin{aligned}
\frac{1}{2 R} \int_{-R}^{R} \operatorname{var}\left(\mathbb{u}_{e s t}(x)\right) d x= & \frac{1}{2 R}\left(\int_{-R}^{R} \sum_{n=-\infty}^{\infty}\left[\left(\frac{1}{|v|} \alpha\left(\frac{\dot{v}}{v}\right) * \tilde{u}\right)(n)(\gamma(x-n))^{2}\right] d x\right) \\
& +\frac{\mu\|\alpha\|_{L^{1}(\mathbb{R})}+\sigma_{r}^{2}}{2 \pi} \int_{\mathbb{R}} \frac{1}{|\hat{\alpha}(\xi v)|^{2}} \mathbb{1}_{[-\pi, \pi]}(\xi) d \xi .
\end{aligned}
$$

Combining (66) and (67)-(68), we obtain

$$
\frac{1}{2 R} \int_{-R}^{R} \operatorname{var}\left(\mathbb{1}_{e s t}(x)\right) d x \stackrel{R \rightarrow \infty}{\longrightarrow} \frac{\mu\|\alpha\|_{L^{1}(\mathbb{R})}+\sigma_{r}^{2}}{2 \pi} \int_{\mathbb{R}} \frac{1}{|\hat{\alpha}(\xi v)|^{2}} \mathbb{1}_{[-\pi, \pi]}(\xi) d \xi .
$$

Hence, combining (52) page 20 and (69), we deduce that, for any $x \in \mathbb{R}$,

$$
\frac{1}{2 R} \int_{-R}^{R} \mathbb{E}\left(\left|\mathbf{w}_{e s t}(x)-u(x)\right|^{2}\right) d x \stackrel{R \rightarrow \infty}{\longrightarrow} \frac{\mu\|\alpha\|_{L^{1}(\mathbb{R})}+\sigma_{r}^{2}}{2 \pi} \int_{\mathbb{R}} \frac{1}{|\hat{\alpha}(\xi v)|^{2}} \mathbb{1}_{[-\pi, \pi]}(\xi) d \xi .
$$

Thus, we proved the following theorem 
Theorem 3.8. (MSE and SNR of the coded exposure method.)

Consider a scene $u(x-v t$ ) of mean $\mu$ (see definition 2.1 page 8 ) that moves at velocity $v$ and let $\sigma_{r}^{2}$ the (finite) variance of the additive (readout) noise. From (70) the MSE of the final crisp image $\mathbb{u}_{\text {est }}$ defined in proposition 3.7 page 19 satisfies

$$
M S E_{\text {flutter }}(\alpha):=\lim _{R \rightarrow+\infty} \frac{1}{2 R} \int_{-R}^{R} \mathbb{E}\left(\left|\mathbf{u}_{\text {est }}(x)-u(x)\right|^{2}\right) d x=\frac{1}{2 \pi} \int_{-\pi}^{\pi} \frac{\mu\|\alpha\|_{L^{1}(\mathbb{R})}+\sigma_{r}^{2}}{|\hat{\alpha}(\xi v)|^{2}} d \xi .
$$

From (51) and (69) the SNR of a coded exposure camera is

$$
S N R_{\text {flutter }}(\alpha):=\frac{\lim _{R \rightarrow+\infty} \frac{1}{2 R} \int_{-R}^{R} \mathbb{E}\left(\mathbb{u}_{e s t}(x)\right) d x}{\sqrt{\lim _{R \rightarrow+\infty} \frac{1}{2 R} \int_{-R}^{R} \operatorname{var}\left(\mathbb{u}_{e s t}(x)\right) d x}}=\frac{\mu}{\sqrt{\frac{1}{2 \pi} \int_{-\pi}^{\pi} \frac{\mu\|\alpha\|_{L^{1}(\mathbb{R})}+\sigma_{r}^{2}}{|\hat{\alpha}(\xi v)|^{2}}} d \xi} .
$$

We now connect the formulae in theorem 3.8 with the existing literature on the coded exposure method. We have that the mean photon emission $\mu$ relates to $\bar{i}_{0}$ in, e.g., [3, section 2]. In addition, from (72), we have that for fixed exposure function $\alpha$ and additive (readout) noise variance $\sigma_{r}^{2}$ the SNR evolves proportionally to $\sqrt{\frac{\mu}{1+\frac{\sigma_{r}^{2}}{\mu}}}$ with the mean photon emission $\mu$. In particular, from (72), if $\sigma_{r}^{2}=0$ and for a fixed $\alpha$ we deduce that the SNR evolves proportionally to $\sqrt{\mu}$ and we retrieve the fundamental theorem of photography. We now turn to the optimization of the coded exposure method. We shall optimize the coded exposure method for given observed scene and sensor. This means that the mean photon emission $\mu$ and additive (readout) noise variance $\sigma_{r}^{2}$ are fixed. Under these conditions, from theorem 3.8 we deduce that the optimization of the coded exposure method boils down to finding an exposure function $\alpha$ that minimizes the MSE given by formula (71) or, equivalently, of that maximizes the SNR given by formula (72). Notice that, to the best of our knowledge, this is the optimization framework considered in the literature on the coded exposure method [1, 3, 2, 5, 17, 29, 27, 34].

Recall that it is equivalent to minimize (71) or to maximize (72) with respect to the exposure function $\alpha$. Therefore, in the sequel we choose w.l.o.g. to use formula (71) and to evaluate the performance of the coded exposure method in terms of MSE. The calculation for the SNR can be immediately deduced. We now summarize the construction of section 3 .

To sum up, in section 3 we have defined the observed samples obs $(n)$ of a coded exposure camera (see definition 3 page 11). We have defined the Shannon-Whittaker interpolated image $o b s(x)$ for any $x \in \mathbb{R}$ (see proposition 3.4 page 16) of the stochastic observed samples obs $(n)$ that are only obtained for $n \in \mathbb{Z}$. We have defined the inverse filter and proved rigorously the existence of $\mathfrak{n}_{e s t}$ i.e., the final crisp image. The final crisp $\mathfrak{a}_{e s t}(x)$ is designed to ensure that $\mathbb{n} \mathbb{u}_{\text {est }}(x)$ is equal to the observable scene $u(x)$ in expectation i.e., $\mathbb{E}\left(\mathbb{u}_{\text {est }}(\cdot)\right)=u(\cdot)$. In addition, this final crisp image $\mathbb{v}_{e s t}$ is obtained from the observed stochastic samples obs $(n)$ (see proposition 3.7 page 19) knowing the exposure function $\alpha$ and the relative camera scene velocity $v$. Theorem 3.8 gives the MSE and SNR of the coded exposure method as a function of the exposure function $\alpha$ (see definition 3 page 11), of the relative camera scene velocity $v$, of the mean photon emission $\mu$ and of the additive noise variance (see definition 2.1 page 8 ).

As an easy application of theorem 3.8, we have the following corollary that provides the MSE of any invertible snapshot, i.e. that satisfies $|v| \Delta t<2$ (see the discussion on page 12) where $\Delta t$ is the exposure time. This corollary will also be needed to compare the coded exposure method and the snapshot, in terms of MSE, in section 4. 


\section{Corollary 3.9. (MSE of a snapshot with an exposure time of $\Delta t$. )}

Consider a scene $u(x-v t$ ) of mean $\mu$ (see definition 2.1 page 8) that moves at velocity $v$ and let $\sigma_{r}^{2}$ be the (finite) variance of the additive (readout) noise. The MSE of a snapshot with exposure time $\Delta t$ is

$$
M S E_{\text {snapshot }}(\Delta t)=\lim _{R \rightarrow+\infty} \frac{1}{2 R} \int_{-R}^{R} \mathbb{E}\left(\left|\mathbb{w}_{\text {est }}(x)-u(x)\right|^{2}\right) d x=\int_{-\pi}^{\pi} \frac{\mu \Delta t+\sigma_{r}^{2}}{\left|\Delta t \operatorname{sinc}\left(\frac{\xi v \Delta t}{2 \pi}\right)\right|^{2}} d \xi .
$$

Proof. The proof is immediate combining (13) page 12 and (71).

We now turn to section 4 that proposes a theoretical evaluation of the gain, in terms of MSE, of the coded exposure method, with respect a snapshot.

\section{An upper bound of performance for coded exposure cameras}

This section study the gain, in terms of MSE, of the coded exposure method, with respect to a snapshot, as a function of the exposure code sampling rate. The study yields to a theoretical bound that is formalized in theorem 4.1 page 28 and corollary 4.2 page 31 . The bound is valid for any exposure code provided $|v| \Delta t \leqslant 1$. (We recall that the exposure code sampling rate $\Delta t$ is defined in definition 3 page 11.) This means that the proposed bound is an upper bound for the gain of any coded exposure camera, provided $|v| \Delta t \leqslant 1$.

We first compute a lower bound for the MSE of coded exposure camera. This lower bound is then used to compute an upper bound for the gain of coded exposure cameras, in terms of MSE, by comparison with the MSE of a well chosen snapshot (standard camera).

We now calculate a lower bound for the MSE of coded exposure cameras. From (71) page 23, we recall that, for any exposure function $\alpha$, we have that

$$
\lim _{T \rightarrow+\infty} \frac{1}{2 R} \int_{-R}^{R} \mathbb{E}\left(\left|\mathbf{u}_{e s t}(x)-u(x)\right|^{2}\right) d x=\int_{-\pi}^{\pi} \frac{\mu\|\alpha\|_{L^{1}(\mathbb{R})}+\sigma_{r}^{2}}{|\hat{\alpha}(\xi v)|^{2}} \frac{d \lambda(\xi)}{2 \pi},
$$

where $d \lambda$ denotes the Lebesgue measure on $(\mathbb{R}, \mathcal{B}(\mathbb{R}))$. From its definition 3 page 11 we have that $\alpha(t) \in[0,1]$ for any $t \in \mathbb{R}$ and that $\alpha \in L^{1}(\mathbb{R})$. Hence, we deduce that $\alpha^{2} \leqslant \alpha$ and that $\int_{\mathbb{R}}|\alpha(x)| d x \geqslant \int_{\mathbb{R}}|\alpha(x)|^{2} d x$. Thus, we derive that $\alpha \in L^{2}(\mathbb{R})$ and from (74) we obtain that

$$
\lim _{R \rightarrow+\infty} \frac{1}{2 R} \int_{-R}^{R} \mathbb{E}\left(\left|\mathbf{u}_{e s t}(x)-u(x)\right|^{2}\right) d x \geqslant \int_{-\pi}^{\pi} \frac{\mu\|\alpha\|_{L^{2}(\mathbb{R})}^{2}+\sigma_{r}^{2}}{|\hat{\alpha}(\xi v)|^{2}} \frac{d \lambda(\xi)}{2 \pi},
$$

for any exposure function. Furthermore, the function $[0,+\infty) \ni x \mapsto x^{2}$ is strictly convex. In addition, we have $\frac{d \lambda([-\pi, \pi])}{2 \pi}=1$. Thus, by Jensen inequality (see, e.g., [20, p. 232]) we obtain that, for any exposure function $\alpha$,

$$
\left(\mu\|\alpha\|_{L^{2}(\mathbb{R})}^{2}+\sigma_{r}^{2}\right) \int_{-\pi}^{\pi} \frac{1}{|\hat{\alpha}(\xi v)|^{2}} \frac{d \lambda(\xi)}{2 \pi} \geqslant\left(\mu\|\alpha\|_{L^{2}(\mathbb{R})}^{2}+\sigma_{r}^{2}\right) \frac{1}{\int_{-\pi}^{\pi}|\hat{\alpha}(\xi v)|^{2} \frac{d \lambda(\xi)}{2 \pi}} .
$$

The strict convexity of $[0,+\infty) \ni x \mapsto x^{2}$ implies that the equality case in (76) is realized when $\xi \mapsto|\hat{\alpha}(\xi v)|^{2}$ is constant say $C>0$ on the interval $[-\pi, \pi]$. By Plancherel formula (146), from the term $\|\alpha\|_{L^{2}(\mathbb{R})}^{2}$ in (76), we deduce that an optimal $\alpha$, if it exists, satisfies $\hat{\alpha}$ supported on the interval $[-\pi|v|, \pi|v|]$. Therefore, we deduce that an optimal $\alpha$, if it exists, satisfies 
$|\hat{\alpha}(\xi)|=C \mathbb{1}_{[-\pi|v|, \pi|v|]}(\xi)$, for some constant $C>0$. Assuming the existence of such a function for any $C$, we can produce a lower bound of (76) by adjusting the constant $C$. The value of the constant $C$ will by computed later on. For example it is necessary that $|\alpha| \leqslant 1$ for a function to be an exposure function (see definition 3 page 11).

We shall now build an exposure code (see definition 3 page 11) such that $|\hat{\alpha}(\xi)|=C \mathbb{1}_{[-\pi|v|, \pi|v|]}(\xi)$ and compute its MSE using theorem 3.8 page 23 . We shall relax the constraint $0 \leqslant \alpha \leqslant 1$ to $|\alpha| \leqslant 1$. The feasible functions of this relaxed problem is thus bigger (in the sense of the inclusion). This means that the bound, in terms of MSE, that we shall obtain is a lower bound for the MSE of coded exposure cameras (see definition 3 page 11). Note that the bound we shall provide applies to finitely supported (finite codes) exposure functions as well as theoretical infinitely supported exposure functions.

From definition 3 page 11, by an easy calculation of a Fourier transform (xv), we deduce that, for any $\xi \in \mathbb{R}$,

$$
|\hat{\alpha}(\xi)|^{2}=\left|\Delta t \operatorname{sinc}\left(\frac{\xi \Delta t}{2 \pi}\right) \sum_{k=-\infty}^{+\infty} \alpha_{k} e^{-i k \xi \Delta t}\right|^{2} \text { and }\|\alpha\|_{L^{2}(\mathbb{R})}^{2}=\Delta t \sum_{k=-\infty}^{+\infty} \alpha_{k}^{2} .
$$

Hence, combining (77) the inequality (76) rewrites as

$$
\int_{-\pi}^{\pi} \frac{\mu \Delta t \sum_{k=-\infty}^{+\infty} \alpha_{k}^{2}+\sigma_{r}^{2}}{\left|\Delta t \operatorname{sinc}\left(\frac{\xi v \Delta t}{2 \pi}\right) \sum_{k=-\infty}^{+\infty} \alpha_{k} e^{-i k \xi v \Delta t}\right|^{2}} \frac{d \lambda(\xi)}{2 \pi} \geqslant \frac{\mu \Delta t \sum_{k=-\infty}^{+\infty} \alpha_{k}^{2}+\sigma_{r}^{2}}{\int_{-\pi}^{\pi}\left|\Delta t \operatorname{sinc}\left(\frac{\xi v \Delta t}{2 \pi}\right) \sum_{k=-\infty}^{+\infty} \alpha_{k} e^{-i k \xi v \Delta t}\right|^{2} \frac{d \lambda(\xi)}{2 \pi}} .
$$

Note that the LHS of (78) is nothing but the MSE of a coded exposure camera with exposure code $\left(\alpha_{k}\right)_{k}$. By a change of variable the RHS of (78) rewrites as

$\frac{\mu \Delta t \sum_{k=-\infty}^{+\infty} \alpha_{k}^{2}+\sigma_{r}^{2}}{\int_{-\pi}^{\pi}\left|\Delta t \operatorname{sinc}\left(\frac{\xi v \Delta t}{2 \pi}\right) \sum_{k=-\infty}^{+\infty} \alpha_{k} e^{-i k \xi v \Delta t}\right|^{2} \frac{d \lambda(\xi)}{2 \pi}}=\frac{\mu \Delta t \sum_{k=-\infty}^{+\infty} \alpha_{k}^{2}+\sigma_{r}^{2}}{\frac{1}{2 \pi|v| \Delta t} \int_{-\pi|v| \Delta t}^{\pi|v| \Delta t}\left|\Delta t \operatorname{sinc}\left(\frac{\xi}{2 \pi}\right) \sum_{k=-\infty}^{+\infty} \alpha_{k} e^{-i k \xi v \Delta t}\right|^{2} d \xi}$.

As we have just seen, a lower bound for the MSE of the coded exposure method can obtained by finding an exposure code such that $\xi \mapsto\left|\Delta t \operatorname{sinc}\left(\frac{\xi v \Delta t}{2 \pi}\right) \sum_{k=-\infty}^{+\infty} \alpha_{k} e^{-i k \xi v \Delta t}\right|$ is constant on $[-\pi, \pi]$. We shall now construct such a code. The construction is based on Fourier series. In other words, we shall identify the $a_{k}$ with the Fourier series coefficient of a well chosen function. As soon as $|v| \Delta t \leqslant 1$, we have $[-\pi|v| \Delta t, \pi|v| \Delta t] \subset[-\pi, \pi]$. Thus, provided $|v| \Delta t \leqslant 1$ we have

$$
\begin{aligned}
& \frac{1}{2 \pi|v| \Delta t} \int_{-\pi|v| \Delta t}^{\pi|v| \Delta t}\left|\Delta t \operatorname{sinc}\left(\frac{\xi}{2 \pi}\right) \sum_{k=-\infty}^{+\infty} \alpha_{k} e^{-i k \xi}\right|^{2} d \xi \\
& =\frac{1}{2 \pi|v| \Delta t} \int_{-\pi}^{\pi}\left|\Delta t \operatorname{sinc}\left(\frac{\xi}{2 \pi}\right) \sum_{k=-\infty}^{+\infty} \alpha_{k} e^{-i k \xi}\right|^{2} \mathbb{1}_{[-\pi|v| \Delta t, \pi|v| \Delta t]}(\xi) d \xi .
\end{aligned}
$$

Provided $|v| \Delta t \leqslant 1$, from the Fourier series decomposition (xix), given all the $\alpha_{k}$ we deduce that $\sum_{k=-\infty}^{+\infty} \alpha_{k} e^{-i k \xi}$ is the Fourier series of the $2 \pi$ periodic function that writes as

$$
\xi \mapsto \sum_{k=-\infty}^{+\infty} \alpha_{k} e^{i k \xi} \mathbb{1}_{[-\pi|v| \Delta t, \pi|v| \Delta t]}(\xi)
$$


Conversely, provided $|v| \Delta t \leqslant 1$, consider the $2 \pi$ periodic and even function $f: \mathbb{R} \rightarrow \mathbb{R}$ defined by

$$
f(\xi)=\frac{C}{\Delta t \operatorname{sinc}\left(\frac{\xi}{2 \pi}\right)} \mathbb{1}_{[-\pi|v| \Delta t, \pi|v| \Delta t]}(\xi) \text { for any } \xi \in[-\pi, \pi],
$$

where $C$ a positive constant that we shall fix shortly. Provided $|v| \Delta t \leqslant 1$ the function $f$ defined in (82) is well defined and $f \in L^{2}([-\pi, \pi])$. Therefore, $f$ has a Fourier series decomposition (xix) and $f(\xi)=\sum_{k=-\infty}^{+\infty} c_{k}(f) e^{i k \xi}$ for, in particular, any $\xi \in[-\pi, \pi]$. In addition, since from its definition (82) we have $f \in L^{2}([-\pi, \pi])$ is even, we have $c_{k}(f) \in \mathbb{R}$ and $\left(c_{k}(f)\right)_{k} \in \ell^{2}(\mathbb{Z})$. Thus, we have

$$
f(\xi)=\sum_{k=-\infty}^{+\infty} c_{k}(f) e^{-i k \xi} \text { for any } \xi \in[-\pi, \pi] .
$$

Thus, combining (82) and (83) we obtain

$$
\sum_{k=-\infty}^{+\infty} c_{k}(f) e^{-i k \xi}=\frac{C \mathbb{1}_{[-\pi|v| \Delta t, \pi|v| \Delta t]}(\xi)}{\Delta t \operatorname{sinc}\left(\frac{\xi}{2 \pi}\right)} \text { for any } \xi \in[-\pi, \pi] .
$$

From now on we choose $a_{k}:=c_{k}(f)$ for any $k \in \mathbb{Z}$. Combining (80)-(81) and (84) we have

$$
\frac{1}{2 \pi|v| \Delta t} \int_{-\pi|v| \Delta t}^{\pi|v| \Delta t}\left|\Delta t \operatorname{sinc}\left(\frac{\xi}{2 \pi}\right) \sum_{k=-\infty}^{+\infty} c_{k}(f) e^{-i k \xi}\right|^{2} d \xi=\frac{2 \pi|v| \Delta t C^{2}}{2 \pi|v| \Delta t}=C^{2} .
$$

Hence, combining (76) page 24 and (85) we obtain

$$
\frac{\mu \Delta t \sum_{k=-\infty}^{+\infty}\left(c_{k}(f)\right)^{2}+\sigma_{r}^{2}}{\int_{-\pi}^{\pi}\left|\Delta t \operatorname{sinc}\left(\frac{\xi v \Delta t}{2 \pi}\right) \sum_{k=-\infty}^{+\infty} c_{k}(f) e^{-i k \xi v \Delta t}\right|^{2} \frac{d \lambda(\xi)}{2 \pi}}=\frac{\mu \Delta t \sum_{k=-\infty}^{+\infty}\left(c_{k}(f)\right)^{2}+\sigma_{r}^{2}}{C^{2}} .
$$

We now fix the value of $C$. From their definitions (xix), for any $k \in \mathbb{Z}$, we have

$$
\left|c_{k}(f)\right| \leqslant \frac{1}{2 \pi} \int_{-\pi}^{\pi} \frac{C}{\Delta t \operatorname{sinc}\left(\frac{\xi}{2 \pi}\right)} \mathbb{1}_{[-\pi|v| \Delta t, \pi|v| \Delta t]}(\xi)=\frac{1}{2 \pi \Delta t} \int_{-\pi|v| \Delta t}^{\pi|v| \Delta t} \frac{C}{\operatorname{sinc}\left(\frac{\xi}{2 \pi}\right)} d \xi .
$$

From (86) we would like the constant $C$ to be as large as possible to get our lower bound. However, from (87) we deduce that we need $C \leqslant \frac{1}{\frac{1}{2 \pi \Delta t} \int_{-\pi|v| \Delta t}^{\pi|v|} \frac{C}{\operatorname{sinc}\left(\frac{\xi}{2 \pi}\right)} d \xi}$ in order to ensure that $\left|c_{k}(f)\right| \leqslant 1$ for any $k \in \mathbb{Z}$. Therefore, we deduce that the choice

$$
C=\frac{1}{\frac{|v|}{2 \pi} \int_{-\pi}^{\pi} \frac{1}{\operatorname{sinc}\left(\frac{\xi v \Delta t}{2 \pi}\right)} d \xi}
$$

in the definition of $f(82)$ is enough to provide a lower bound to the MSE of coded exposure cameras.

We recall that (78) is valid for any exposure code provided $|v| \Delta t \leqslant 1$. In addition, provided $|v| \Delta t \leqslant 1$, we can choose the $a_{k}$ given by the Fourier series decomposition (xix) of $f$ defined in (82) with the constant $C$ given by (88). Furthermore, this choice provides a lower bound of (78) page 25. Therefore, combining (78) and (86) we deduce that

$$
\int_{-\pi}^{\pi} \frac{\mu \Delta t \sum_{k=-\infty}^{+\infty} \alpha_{k}^{2}+\sigma_{r}^{2}}{\left|\operatorname{sinc}\left(\frac{\xi v \Delta t}{2 \pi}\right) \sum_{k=-\infty}^{+\infty} \alpha_{k} e^{-i k \xi v \Delta t}\right|^{2}} \frac{d \lambda(\xi)}{2 \pi} \geqslant \frac{\mu \Delta t \sum_{k=-\infty}^{+\infty}\left(c_{k}(f)\right)^{2}+\sigma_{r}^{2}}{C^{2}}
$$


for any exposure function $\alpha$. Hence, combining (75) page 24, (77) and (89), we obtain

$$
\lim _{T \rightarrow+\infty} \frac{1}{2 T} \int_{-T}^{T} \mathbb{E}\left(\left|\mathbf{u}_{e s t}(x)-u(x)\right|^{2}\right) d x \geqslant \frac{\mu \Delta t \sum_{k=-\infty}^{+\infty}\left(c_{k}(f)\right)^{2}+\sigma_{r}^{2}}{C^{2}}
$$

for any exposure code. It remains to evaluate $\sum_{k=-\infty}^{+\infty}\left(c_{k}(f)\right)^{2}$. From its definition (82) page 26 $f$ is $2 \pi$ periodic and we have $f \in L^{2}([-\pi, \pi])$. Therefore, by Parseval formula (147) and (82) page 26 we have

$$
\sum_{k=-\infty}^{+\infty}\left|c_{k}(f)\right|^{2}=\frac{1}{2 \pi} \int_{-\pi}^{\pi}\left|\frac{C \mathbb{1}_{[-\pi|v| \Delta t, \pi|v| \Delta t]}(\xi)}{\Delta t \operatorname{sinc}\left(\frac{\xi}{2 \pi}\right)}\right|^{2} d \xi=\frac{C^{2}}{2 \pi \Delta t^{2}} \int_{-\pi|v| \Delta t}^{\pi|v| \Delta t} \frac{1}{\operatorname{sinc}^{2}\left(\frac{\xi}{2 \pi}\right)} d \xi .
$$

Thus, from (91) we have

$$
\sum_{k=-\infty}^{+\infty}\left|c_{k}(f)\right|^{2}=\frac{C^{2}|v|}{2 \pi \Delta t} \int_{-\pi}^{\pi} \frac{1}{\operatorname{sinc}^{2}\left(\frac{\xi|v| \Delta t}{2 \pi}\right)} d \xi
$$

On the one hand, from (92), we have

$$
\frac{\mu \Delta t \sum_{k=-\infty}^{+\infty}\left(c_{k}(f)\right)^{2}}{C^{2}}=\frac{1}{C^{2}} \mu \Delta t \frac{C^{2}|v|}{2 \pi \Delta t} \int_{-\pi}^{\pi} \frac{1}{\operatorname{sinc}^{2}\left(\frac{\xi|v| \Delta t}{2 \pi}\right)} d \xi=\frac{\mu|v|}{2 \pi} \int_{-\pi}^{\pi} \frac{1}{\operatorname{sinc}^{2}\left(\frac{\xi|v| \Delta t}{2 \pi}\right)} d \xi .
$$

On the other hand, from (88), we have

$$
\frac{\sigma_{r}^{2}}{C^{2}}=\sigma_{r}^{2}\left(\frac{|v|}{2 \pi} \int_{-\pi}^{\pi} \frac{1}{\operatorname{sinc}\left(\frac{\xi v \Delta t}{2 \pi}\right)} d \xi\right)^{2}
$$

Thus, combining (93) and (94) we deduce that

$$
\frac{\mu \Delta t \sum_{k=0}^{L-1}\left(c_{k}(f)\right)^{2}+\sigma_{r}^{2}}{C^{2}}=\frac{\mu|v|}{2 \pi} \int_{-\pi}^{\pi} \frac{1}{\operatorname{sinc}^{2}\left(\frac{\xi|v| \Delta t}{2 \pi}\right)} d \xi+\sigma_{r}^{2}\left(\frac{|v|}{2 \pi} \int_{-\pi}^{\pi} \frac{1}{\operatorname{sinc}\left(\frac{\xi v \Delta t}{2 \pi}\right)} d \xi\right)^{2} .
$$

Therefore, from (95) we deduce that

$$
\frac{\mu \Delta t \sum_{k=0}^{L-1}\left(c_{k}(f)\right)^{2}+\sigma_{r}^{2}}{C^{2}}=|v|\left[\frac{\mu}{2 \pi} \int_{-\pi}^{\pi} \frac{d \xi}{\operatorname{sinc}^{2}\left(\frac{\xi|v| \Delta t}{2 \pi}\right)}+\sigma_{r}^{2}|v|\left(\frac{1}{2 \pi} \int_{-\pi}^{\pi} \frac{d \xi}{\operatorname{sinc}\left(\frac{\xi v \Delta t}{2 \pi}\right)}\right)^{2}\right]
$$

Thus, combining (90) and (96) we obtain

$$
\lim _{T \rightarrow+\infty} \frac{1}{2 T} \int_{-T}^{T} \mathbb{E}\left(\left|\mathbf{u}_{e s t}(x)-u(x)\right|^{2}\right) d x \geqslant|v|\left[\frac{\mu}{2 \pi} \int_{-\pi}^{\pi} \frac{d \xi}{\operatorname{sinc}^{2}\left(\frac{\xi|v| \Delta t}{2 \pi}\right)}+\sigma_{r}^{2}|v|\left(\frac{1}{2 \pi} \int_{-\pi}^{\pi} \frac{d \xi}{\operatorname{sinc}\left(\frac{\xi v \Delta t}{2 \pi}\right)}\right)^{2}\right],
$$

for any exposure code. Thus, we proved 
Theorem 4.1. (A lower bound for the MSE of coded exposure cameras.)

Consider a scene $u(x-v t$ ) of mean $\mu$ (see definition 2.1 page 8) that moves at velocity $v$ and let $\sigma_{r}^{2}$ the (finite) variance of the additive (readout) noise. The MSE of any coded exposure camera satisfies

$$
M S E_{\text {any flutter }}(\alpha) \geqslant|v|\left[\frac{\mu}{2 \pi} \int_{-\pi}^{\pi} \frac{d \xi}{\operatorname{sinc}^{2}\left(\frac{\xi|v| \Delta t}{2 \pi}\right)}+\sigma_{r}^{2}|v|\left(\frac{1}{2 \pi} \int_{-\pi}^{\pi} \frac{d \xi}{\operatorname{sinc}\left(\frac{\xi v \Delta t}{2 \pi}\right)}\right)^{2}\right],
$$

as soon as $|v| \Delta t \leqslant 1$.

In order to evaluate the maximal theoretical "gain" of coded exposure camera we need to compare the bound in terms of MSE given in theorem 4.1 with the MSE of a snapshot. We now compute the MSE of a well chosen snapshot. From the definition of the sinc function (xvii) we have

$$
\frac{1}{2 \pi} \int_{-\pi}^{\pi} \frac{\mu \Delta t+\sigma_{r}^{2}}{\left|\Delta t \operatorname{sinc}\left(\frac{\xi v \Delta t}{2 \pi}\right)\right|^{2}} d \xi=\frac{1}{2 \pi} \int_{-\pi}^{\pi} \frac{\mu \Delta t+\sigma_{r}^{2}}{\left|\Delta t \frac{\sin \left(\pi \frac{\xi v \Delta t}{2 \pi}\right)}{\pi \frac{\xi \Delta t}{2 \pi}}\right|^{2}} d \xi=\frac{1}{2 \pi} \int_{-\pi}^{\pi} \frac{\mu \Delta t+\sigma_{r}^{2}}{\left|\Delta t \frac{\sin \left(\frac{\xi v \Delta t}{2}\right)}{\frac{\xi \Delta \Delta t}{2}}\right|^{2}} d \xi .
$$

Hence, from (98) we have

$$
\frac{1}{2 \pi} \int_{-\pi}^{\pi} \frac{\mu \Delta t+\sigma_{r}^{2}}{\left|\Delta t \operatorname{sinc}\left(\frac{\xi v \Delta t}{2 \pi}\right)\right|^{2}} d \xi=\frac{1}{2 \pi} \int_{-\pi}^{\pi} \frac{\mu \Delta t+\sigma_{r}^{2}}{\left|2 \Delta t \frac{\sin \left(\frac{\xi v \Delta t}{2}\right)}{\xi v \Delta t}\right|^{2}} d \xi=\frac{1}{2 \pi} \int_{-\pi}^{\pi} \frac{\mu \Delta t+\sigma_{r}^{2}}{\left|2 \frac{\sin \left(\frac{\xi v \Delta t}{2}\right)}{\xi v}\right|^{2}} d \xi
$$

Thus, from (99) we obtain

$$
\frac{1}{2 \pi} \int_{-\pi}^{\pi} \frac{\mu \Delta t+\sigma_{r}^{2}}{\left|\Delta t \operatorname{sinc}\left(\frac{\xi v \Delta t}{2 \pi}\right)\right|^{2}} d \xi=\frac{1}{2 \pi} \int_{-\pi}^{\pi} \frac{\mu \Delta t+\sigma_{r}^{2}}{\left|2 \frac{\sin \left(\frac{\xi v \Delta t}{\xi v}\right)}{\xi v}\right|^{2}} d \xi=\frac{\mu \Delta t+\sigma_{r}^{2}}{2 \pi} \int_{-\pi}^{\pi}\left|\frac{\xi v}{2 \sin \left(\frac{\xi v \Delta t}{2}\right)}\right|^{2} d \xi .
$$

Therefore, from (100) we deduce that

$$
\frac{1}{2 \pi} \int_{-\pi}^{\pi} \frac{\mu \Delta t+\sigma_{r}^{2}}{\left|\Delta t \operatorname{sinc}\left(\frac{\xi v \Delta t}{2 \pi}\right)\right|^{2}} d \xi=|v| \frac{\mu|v| \Delta t+|v| \sigma_{r}^{2}}{2 \pi} \int_{-\pi}^{\pi}\left|\frac{\xi}{2 \sin \left(\frac{\xi v \Delta t}{2}\right)}\right|^{2} d \xi
$$

Thus, combining (73) page 24 and (101), we deduce that the MSE of a snapshot with exposure time equal to $\Delta t:=\frac{1}{|v|}$ is

$$
\operatorname{MSE}_{\text {snapshot }}(\Delta t)=|v|\left(\mu+|v| \sigma_{r}^{2}\right) \frac{1}{2 \pi} \int_{-\pi}^{\pi}\left|\frac{\xi}{2 \sin \left(\frac{\xi}{2}\right)}\right|^{2} d \xi
$$

In addition the optimal snapshot, if it exists has, by definition, a MSE lower or equal to the one in equation (102). In other words, (102) provides an upper bound for the MSE of the optimal snapshot. We now compare the MSE of the coded exposure method and of this snapshot. 
We have

$$
\begin{aligned}
& |v|\left[\frac{\mu}{2 \pi} \int_{-\pi}^{\pi} \frac{d \xi}{\operatorname{sinc}^{2}\left(\frac{\xi|v| \Delta t}{2 \pi}\right)}+\sigma_{r}^{2}|v|\left(\frac{1}{2 \pi} \int_{-\pi}^{\pi} \frac{d \xi}{\operatorname{sinc}\left(\frac{\xi v \Delta t}{2 \pi}\right)}\right)^{2}\right] \\
& =|v|\left(\frac{1}{2 \pi} \int_{-\pi}^{\pi} \frac{d \xi}{\operatorname{sinc}\left(\frac{\xi v \Delta t}{2 \pi}\right)}\right)^{2}\left[\mu \frac{\frac{1}{2 \pi} \int_{-\pi}^{\pi} \frac{d \xi}{\operatorname{sinc}^{2}\left(\frac{\xi|v| \Delta t}{2 \pi}\right)}}{\left(\frac{1}{2 \pi} \int_{-\pi}^{\pi} \frac{d \xi}{\operatorname{sinc}\left(\frac{\xi v \Delta t}{2 \pi}\right)}\right)^{2}}+\sigma_{r}^{2}|v|\right] .
\end{aligned}
$$

In addition, by Jensen inequality (see, e.g., [20, p. 232]), we have

$$
\left(\frac{1}{2 \pi} \int_{-\pi}^{\pi} \frac{d \xi}{\operatorname{sinc}\left(\frac{\xi v \Delta t}{2 \pi}\right)}\right)^{2} \leqslant \frac{1}{2 \pi} \int_{-\pi}^{\pi} \frac{d \xi}{\operatorname{sinc}^{2}\left(\frac{\xi|v| \Delta t}{2 \pi}\right)}
$$

Hence, from (105) we deduce that

$$
\frac{\frac{1}{2 \pi} \int_{-\pi}^{\pi} \frac{d \xi}{\operatorname{sinc}^{2}\left(\frac{\xi|v| \Delta t}{2 \pi}\right)}}{\left(\frac{1}{2 \pi} \int_{-\pi}^{\pi} \frac{d \xi}{\operatorname{sinc}\left(\frac{\xi v \Delta t}{2 \pi}\right)}\right)^{2}} \geqslant 1
$$

Thus, combining (103)-(104) and (106) we deduce that

$|v|\left[\frac{\mu}{2 \pi} \int_{-\pi}^{\pi} \frac{d \xi}{\operatorname{sinc}^{2}\left(\frac{\xi|v| \Delta t}{2 \pi}\right)}+\sigma_{r}^{2}|v|\left(\frac{1}{2 \pi} \int_{-\pi}^{\pi} \frac{d \xi}{\operatorname{sinc}\left(\frac{\xi v \Delta t}{2 \pi}\right)}\right)^{2}\right] \geqslant|v|\left(\frac{1}{2 \pi} \int_{-\pi}^{\pi} \frac{d \xi}{\operatorname{sinc}\left(\frac{\xi v \Delta t}{2 \pi}\right)}\right)^{2}\left[\mu+\sigma_{r}^{2}|v|\right]$.

Therefore, combining (97) page 28 and (107) we deduce that the MSE of any coded exposure camera satisfies

$$
\lim _{R \rightarrow+\infty} \frac{1}{2 R} \int_{-R}^{R} \mathbb{E}\left(\left|\mathbf{u}_{e s t}(x)-u(x)\right|^{2}\right) d x \geqslant|v|\left(\frac{1}{2 \pi} \int_{-\pi}^{\pi} \frac{d \xi}{\operatorname{sinc}\left(\frac{\xi v \Delta t}{2 \pi}\right)}\right)^{2}\left[\mu+\sigma_{r}^{2}|v|\right] .
$$

Hence, from (108) we deduce that

$$
\frac{1}{\operatorname{MSE}_{\text {fluttter }}(\alpha)} \leqslant \frac{1}{|v|\left(\frac{1}{2 \pi} \int_{-\pi}^{\pi} \frac{d \xi}{\operatorname{sinc}\left(\frac{\xi v \Delta t}{2 \pi}\right)}\right)^{2}\left[\mu+\sigma_{r}^{2}|v|\right]} .
$$

Thus, we obtain

$$
\frac{\operatorname{MSE}_{\text {snapshot }}(\Delta t)}{\operatorname{MSE}_{\text {fluttter }}(\alpha)} \leqslant \frac{|v|\left(\frac{1}{2 \pi} \int_{-\pi}^{\pi}\left|\frac{\xi}{2 \sin \left(\frac{\xi}{2}\right)}\right|^{2} d \xi\right)\left[\mu+|v| \sigma_{r}^{2}\right]}{|v|\left(\frac{1}{2 \pi} \int_{-\pi}^{\pi} \frac{d \xi}{\operatorname{sinc}\left(\frac{\xi v \Delta t}{2 \pi}\right)}\right)^{2}\left[\mu+\sigma_{r}^{2}|v|\right]} .
$$

Consequently, from (110) we have

$$
\frac{\operatorname{MSE}_{\text {snapshot }}(\Delta t)}{\operatorname{MSE}_{\text {fluttter }}(\alpha)} \leqslant \frac{\left(\frac{1}{2 \pi} \int_{-\pi}^{\pi}\left|\frac{\xi}{2 \sin \left(\frac{\xi}{2}\right)}\right|^{2} d \xi\right)}{\left(\frac{1}{2 \pi} \int_{-\pi}^{\pi} \frac{d \xi}{\operatorname{sinc}\left(\frac{\xi v \Delta t}{2 \pi}\right)}\right)^{2}}
$$


Thus, we proved 
Corollary 4.2. (Upper bound of any coded exposure camera in terms of MSE with respect to a snapshot)

Consider a scene $u(x-v t)$ of mean $\mu$ (see definition 2.1 page 8) that moves at velocity $v$ and let $\sigma_{r}^{2}$ the (finite) variance of the additive (readout) noise. The gain in terms of MSE of any coded exposure camera with respect to a snapshot with exposure time equal to $\Delta t:=\frac{1}{|v|}$ is from (111) satisfies

$$
\frac{M S E_{\text {snapshot }}(\Delta t)}{M S E_{\text {fluttter }}(\alpha)} \leqslant \frac{\left(\frac{1}{2 \pi} \int_{-\pi}^{\pi}\left|\frac{\xi}{2 \sin \left(\frac{\xi}{2}\right)}\right|^{2} d \xi\right)}{\left(\frac{1}{2 \pi} \int_{-\pi}^{\pi} \frac{d \xi}{\operatorname{sinc}\left(\frac{\xi v \Delta t}{2 \pi}\right)}\right)^{2}}
$$

for any exposure function $\alpha$ that satisfies $|v| \Delta t \leqslant 1$.

We now depict, in figure 2, the upper bound of corollary 4.2 varying the quantity $|v| \Delta t$. Note that the quantity $|v| \Delta t$ is inversely proportional to the temporal frequency sampling of the exposure code. Note that the curve is an upper bound. Thus, the actual gain of the coded exposure method is below this curve.

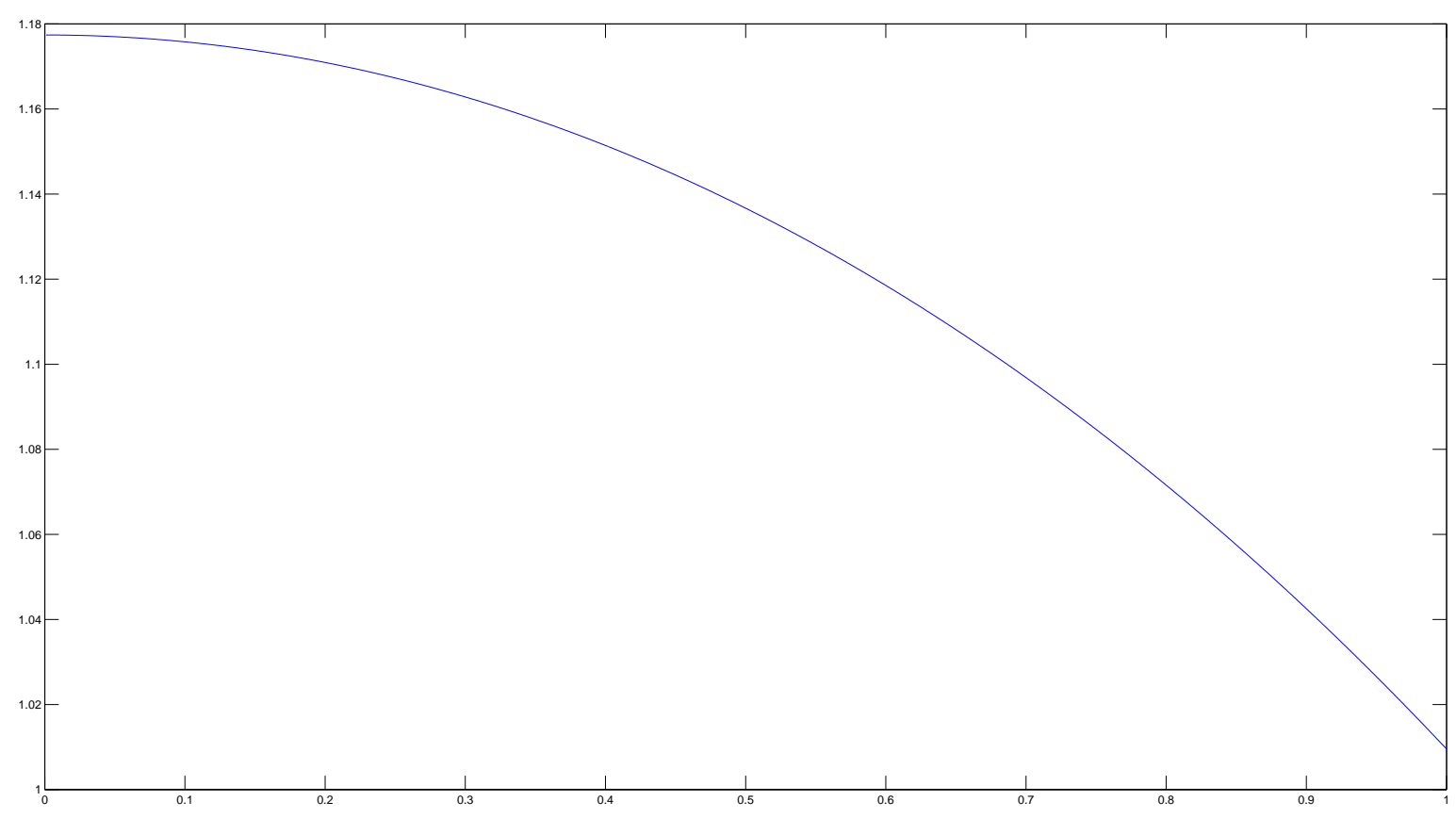

Figure 2: This figure depicts the upper bound proved in corollary 4.2. The $x$-axis represents the quantity $|v| \Delta t$ that is inverse proportional to the frequency sampling of the exposure function. The $x$-axis varies in the interval [0,1] because corollary 4.2 page 31 is valid in this range. The $y$-axis represents the upper bound of the gain, in terms of root mean square error, of the flutter with respect to a snapshot with an exposure time $\Delta t=\frac{1}{1 v}$. Note that the curve is an upper bound. Thus, the actual gain of the coded exposure method is below this curve.

We now illustrate numerically corollary 4.2 in figure 3 and table 1.

\section{Conclusion}



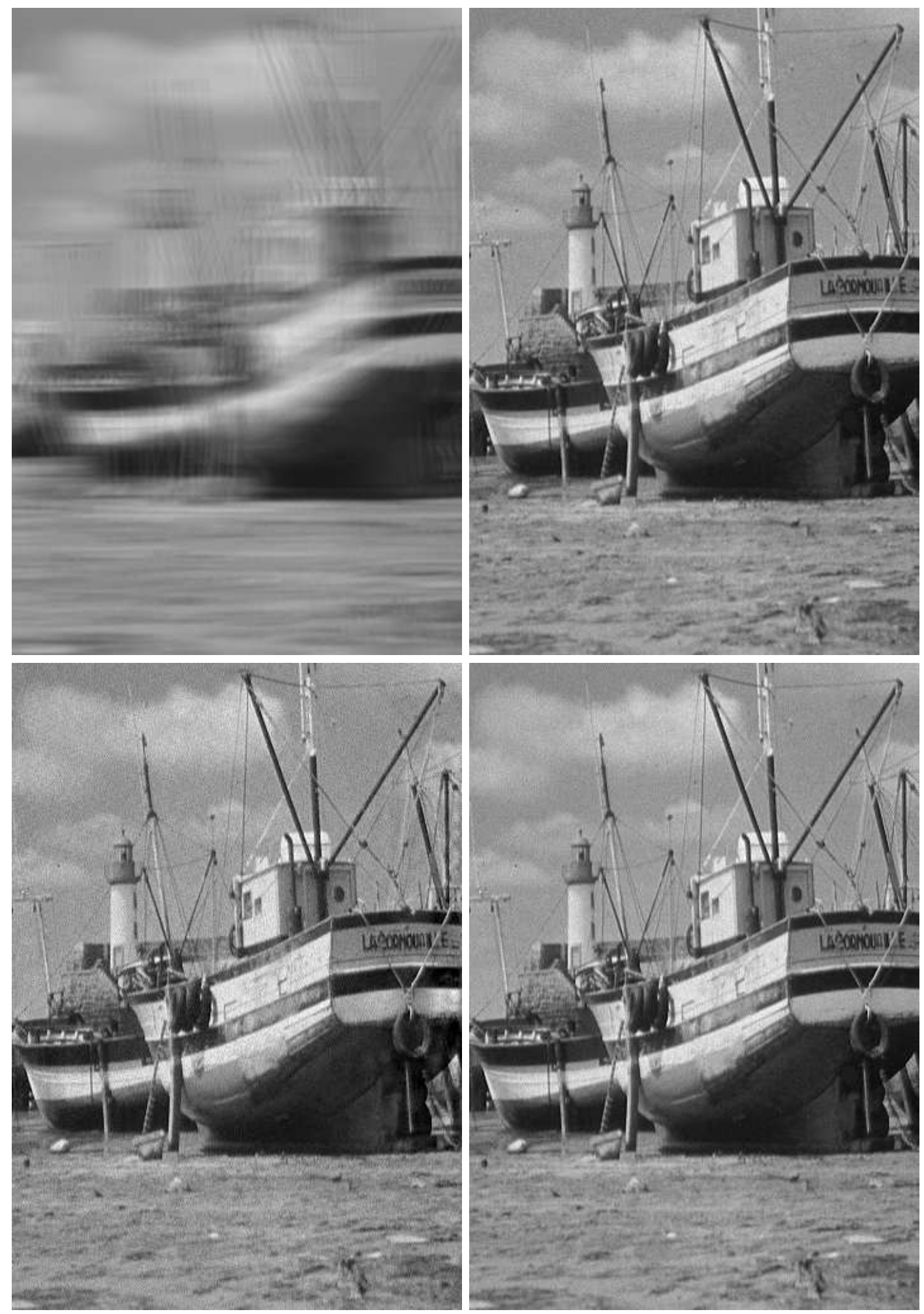

Figure 3: In this experiment, we assume that the scene $s$ moves at velocity $v=1$ pixel per second. The additive (readout) noise is Gaussian with a standard deviation equal to 10 . We also assume that the scene emits 625 photons per seconds (for other values see table 1 pagee 33 ). On the top left panel: the observed image using the Agrawal, Raskar et al. code [38, 39]. On the top right panel: the observed image for snapshot an exposure time of 1 second, i.e., the blur support is 1 pixel. On the bottom left panel: the reconstructed image for the Agrawal, Raskar et al. code $[38,39]$. On the bottom right panel: the reconstructed image for the snapshot (blur support of 1 pixel). This means that for ${ }_{2}$ the Agrawal, Raskar et al. code the blur has a support of 52 pixels. In other words, this code permits to increase the exposure time by a factor 52 compared to the snapshot. The RMSE using the Agrawal, Raskar et al. code is equal to 9.84. The RMSE of the snapshot is equal to 5.96. We refer to table 1 for different values of mean photon count and additive (readout) noise variance. This simulation is based on a variant of [47]. 
Table 1: This table provides the evolution of the RMSE varying the intensity of the mean photon emission for a fixed additive (readout) noise variance. The additive (readout) noise is Gaussian with variance $\sigma_{r}^{2}=100$. A mean photon count of $k$ means that the camera collects $k$ photons if it integrates 1 second. The scene moves, w.l.o.g. at velocity $v=1$ pixel per second. The snapshot integrates during 1 second. The exposure code used is the Agrawal, Raskar et al. code $[38,39]$. This code permits to collect more photons than the snapshot but the deconvolution kernel is more severe than the deconvolution kernel of the snapshot.

\begin{tabular}{|c|c|c|c|c|c|c|c|c|c|}
\hline $\begin{array}{c}\text { Mean photon } \\
\text { count per second }\end{array}$ & 36 & 64 & 100 & 225 & 625 & 1200 & 2500 & 4900 & $10^{4}$ \\
\hline $\begin{array}{c}\text { Readout noise } \\
\text { variance } \sigma_{r}^{2}\end{array}$ & 100 & 100 & 100 & 100 & 100 & 100 & 100 & 100 & 100 \\
\hline RMSE flutter & 42.4 & 31.83 & 25.09 & 16.52 & 9.84 & 7.12 & 4.94 & 3.57 & 2.49 \\
RMSE snapshot & 38.75 & 25.40 & 18.44 & 10.88 & 5.96 & 4.22 & 2.89 & 2.09 & 1.47 \\
\hline
\end{tabular}

We have proposed a mathematical model of coded exposure cameras. The model includes the Poisson photon (shot) noise and any additive readout noise of finite variance. The model is based on the Shannon-Whittaker framework that assumes band limited images. This formalism has allowed us to give closed formulae for the Mean Square Error and Signal to Noise Ratio of coded exposure cameras. In addition, we have given an explicit formula that gives an absolute upper bound for the gain of any coded exposure cameras, in terms of Mean Square Error, with respect to a snapshot. The calculations take into account for the whole imaging chain that includes Poisson photon (shot) noise, any additive (readout) noise of finite variance in addition to the deconvolution. There is a rich body of empirical results on the coded exposure method. They suggest higher gains that our formalism allowed us to prove. The discrepancy may come from an imperfect model of our mathematical coded exposure camera. Indeed, our model assumes that the sensor does not saturate, that the relative camera scene velocity is known, that the scene has finite energy and is observed through an optical system that provides a cut off frequency, that the additive (readout) noise has a finite variance, neglects the boundaries effects due to the deconvolution and neglects the quantization effects. How the results change if one has to estimate the velocity is, to the best of our knowledge, an open question.

\section{Acknowledgments}

The author is happy to deeply thanks "les milieux autorisés": Igor Ciril (IPSA, MITE), Jérôme Darbon (CNRS, UCLA, IX-Labs and the "others offices"), Sylvain Peyronnet (Univ. Caen, IX-Labs), Marc Sigelle (Télécom ParisTech), the one who knocks and the Betches family for their careful readings of the drafts. Indeed, their feedbacks have been duly noted. Yohann Tendero and Stanley Osher are supported on the grant ONR No: N000141410683.

\section{A Validity of the one dimensional framework}

Without loss of generality, we express the coordinates of the two dimensional camera scene velocity vector using the sampling grid as Galilean reference frame. Therefore, in this reference frame, the angle $\theta$ between the two dimensional velocity vector and, e.g., the $x$-axis of the sampling grid is known. Consider the rotation re-sampling operator of angle $\theta$ on $L^{2}\left(\mathbb{R}^{2}\right)$. This rotation operator re-sample the image so that the motion is parallel to one of the grid axis, e.g., the $x$-axis. The motion blur is a one dimensional phenomenon. Therefore, we can 
apply the motion blur model to each line of the image parallel to the $x$-axis. Thus, from the mathematical viewpoint, the motion blur is mathematically equivalent to a one dimensional convolution of a exposure function by the one dimensional observed stochastic scene. Indeed, the whole convolution/deconvolution model is applied to each line of the image parallel to the $x$ axis. In addition, the image rotation operator is isometric in $L^{2}\left(\mathbb{R}^{2}\right)$. Therefore, the calculations of MSE and SNR as if the images were one dimensional signals hold for two dimensional images by easy expectation and variance calculations. This means that the theorems that we shall prove in the sequel are valid for two dimensional images.

\section{B It is enough to study only $[-\pi, \pi]$ band limited functions}

This section justifies that it is enough to consider only $[-\pi, \pi]$ band limited functions. As we have seen page 3 , it is enough to model the coded exposure method as if the images were one dimensional signals. Hence, for the sake of the clarity, we can justify this $[-\pi, \pi]$ as if the image was a sound.

For the sake of the clarity, consider momentarily that the deterministic function $f$ represents a sound. (This example is illustrative of our situation because $u$ defined in definition 2.1 page 8 is one dimensional.) Since we consider a sound $f$, we also momentarily consider that the unit of the variable $x$ in $(\mathbf{x v})$ is the second (denoted s). From the definition of $\hat{f}$ (see $(\mathbf{x v})$ ) we deduce that $\xi$ is in $\frac{\mathrm{Hz}}{2 \pi}(\mathrm{Hz}$ denotes Hertz). If $\hat{f}$ satisfies $\hat{f}(\xi)=0$ for any $\xi \in \mathbb{R}$ such that $|\xi|>\pi$, i.e., is $[-\pi, \pi]$ band limited then the maximal frequency of $f$ is $\frac{\pi}{2 \pi}$ in Hz. Therefore, from the Shannon-Whittaker sampling theorem, $f$ is well sampled with one sample every second.

Consider now an arbitrary function $f$ and a positive real number $c$. If $f$ is sampled with one sample every $\frac{1}{c} \mathrm{~s}$ then, from the Shannon-Whittaker sampling theorem, one can recover any $[-c \pi, c \pi]$ band limited functions. This means that $f$ must not contain any frequency above $\frac{\pi c}{2 \pi}$ in Hz. In other words, by diminishing adequately the sampling step one can recover any band limited function. Consider another time unit $\frac{\mathrm{s}}{c}$, and a zoomed version of $f$ defined by $\tilde{f}(\cdot):=$ $f(\dot{\bar{c}})$. As we have seen, if we have access to the samples $\cdots, f\left(\frac{-1}{c}\right), f\left(\frac{0}{c}\right), f\left(\frac{1}{c}\right), f\left(\frac{2}{c}\right), \cdots$ or equivalently that we have access to $\cdots, \tilde{f}(-1), \tilde{f}(0), \tilde{f}(1), \tilde{f}(2), \cdots$ then $f$ and therefore $\tilde{f}$ are well sampled from the Shannon-Whittaker theory point of view. One the one hand, $f$ is $[-c \pi, c \pi]$ band limited with an arbitrary positive constant $c$ that is $f$ is band limited. This means that $\hat{\tilde{f}}(\xi)=0$ as soon as $|c \xi|>|c \pi|$. Therefore, from its definition, $\hat{\tilde{f}}$ is $[-\pi, \pi]$ band limited. In other words, $f$ can be seen as a $[-\pi, \pi]$ band limited function provided we use an adequate time scale, i.e., use an adequate unit to measure the time. This time scale is given by the Shannon-Whittaker sampling theorem and, implicitly, implies that $\xi$ is measured in $\frac{\pi}{2 \pi c} \mathrm{~Hz}$. Therefore, if we assume that the sampling system respects the Shannon-Whittaker theorem we can without loss of generality assume that $f$ is $[-\pi, \pi]$, where the frequencies of $f$ does not

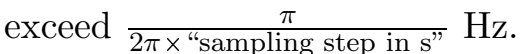

In conclusion, we assume that the couple optical system/sensor array are designed according to the Shannon-Whittaker sampling theory. Therefore, the adequate unit for the distances $x$ is the pixel. With this unit of distance the signal is $[-\pi, \pi]$ band limited, where the frequencies

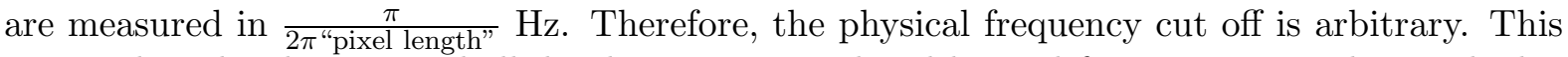
means that the theory we shall develop covers any band limited function. Note that with this model the image has a structure: the structure of a band limited function. Our hypothesis is that, the couple optical system/sensor permits to obtain well sampled signal in the sense of the Shannon-Whittaker sampling theorem. Mathematically, we can, w.l.o.g. assume that the cut off frequency comes from the PSF $g$ as in definition 2.1 page 8 that is $g$ is $[-\pi, \pi]$ band limited. 


\section{Measurability}

We need to prove that the samples are random variables, i.e., measurable functions (see, e.g., [44, p. 168], see also [20, p. 44]). For any $x \in \mathbb{R}$ let the random variables $\eta(x)$ be defined by $\eta(x)$ : $\left(\Omega, \mathcal{T}_{\eta}, \mathbb{P}_{\eta}\right) \rightarrow(\mathbb{R}, \mathcal{B}$ or $(\mathbb{R}))$ where, $\Omega:=\mathbb{R}$ is the sample space, $\mathcal{B}$ or $(\mathbb{R})$ is the Borel sigma algebra on $\mathbb{R}, \mathcal{T}_{\eta}$ is chosen as the smallest tribe on $\Omega$ that makes $\eta(x)$ measurable i.e, $\mathcal{T}_{\eta}=\left\{\eta(k)^{-1} B: B \in\right.$ $\mathcal{B}$ or $(\mathbb{R})\}$. The Poisson random variables are defined on $\mathcal{P}(\lambda):\left(\mathbb{N}, \mathcal{T}_{\mathbb{N}}, \mathbb{P}_{\text {Poisson }(\lambda)}\right) \rightarrow(\mathbb{R}, \mathcal{B}$ or $(\mathbb{R}))$, where $\mathcal{T}_{\mathbb{N}}$ is the smallest sigma algebra (in the sense of the inclusion) that contains $\mathbb{N}$. Assume that at a pixel sensor centered at $x \in \mathbb{R}$ we have $\int_{t_{1}}^{t_{2}} u(x-v t) d t=\lambda$. From equation (9) and for any $x \in \mathbb{R}$, obs $(x)$ is the sum of a Poisson and of a sensor readout $\eta(x)$ random variables $\mathcal{P}(\lambda)+\eta(x):(\Omega, \mathcal{T}, \mathbb{P}) \rightarrow(\mathbb{R}, \mathcal{B}$ or $(\mathbb{R}))$. It is therefore measurable with the sigma algebra $\mathcal{T}=\left\{(\mathcal{P}(\lambda)+\eta(k))^{-1} B: B \in \mathcal{B}\right.$ or $\left.(\mathbb{R})\right\}$.

\section{Proof of lemma 3.2 page 13}

From its definition 2.1 page 8 the deterministic function $\tilde{u}=u-\mu$ is $[-\pi, \pi]$ band limited. Therefore, we deduce that the function $\mathbb{R} \ni x \mapsto\left(\frac{1}{|v|} \alpha(\dot{\bar{v}}) * \tilde{u}\right)(x)$ is $[-\pi, \pi]$ band limited. In addition, since $u(\cdot)=\tilde{u}(\cdot)+\mu$ (see definition 2.1 page 8 ) we deduce that, for any $x \in \mathbb{R},(15)$ holds. We now prove that $u$ is uniformly bounded.

From the remark page 11 we have $\alpha \in L^{1}(\mathbb{R})$. Recall that from definition 2.1 page 8 we have $\tilde{u} \in L^{1}(\mathbb{R})$. Hence, from Young inequality (see, e.g., [25, p. 525]) we deduce that $\left(\frac{1}{|v|} \alpha(\dot{v}) * \tilde{u}\right) \in L^{1}(\mathbb{R})$. Therefore, by Riemann-Lebesgue theorem (see e.g., [23, prop. 2.1]) we have that $\mathbb{R} \ni \xi \mapsto \mathcal{F}(\alpha(\dot{\bar{v}}) * \tilde{u})(\xi)$ is continuous. In addition, from definition 2.1 page 8 we deduce that $\tilde{u}$ is band limited. Therefore, $\mathbb{R} \ni \xi \mapsto \mathcal{F}\left(\frac{1}{|v|} \alpha(\dot{\bar{v}}) * \tilde{u}\right)(\xi)$ is compactly supported. Hence, we deduce that $\mathcal{F}\left(\frac{1}{|v|} \alpha(\dot{\bar{v}}) * \tilde{u}\right) \in L^{1}(\mathbb{R})$. Thus, by Riemann-Lebesgue theorem we have that $\frac{1}{|v|} \alpha(\dot{\dot{v}}) * \tilde{u}$ is uniformly bounded. It follows from (15) that $\frac{1}{|v|} \alpha(\dot{\dot{v}}) * u$ is uniformly bounded.

\section{E Proof of lemma 3.5 page 17}

Consider the $2 \pi$ periodic function $g: \mathbb{R} \rightarrow \mathbb{C}$ defined as

$$
g(\xi):=\hat{\gamma}(\xi) \text { for any } \xi \in[-\pi, \pi)
$$

and the Dirichlet kernel $D_{n}(\xi)=\frac{1}{2 \pi} \sum_{k=-n}^{n} e^{i k \xi}$.

From its definition 3 page 11 we have $\alpha \in L^{1}(\mathbb{R})$. Therefore, by Riemann-Lebesque (see e.g., [23, prop. 2.1]) we have that $\hat{\alpha}$ is continuous. In addition, we assumed that $\hat{\alpha}(\xi v) \neq 0$ for any $\xi \in[-\pi, \pi]$. From their definitions (39) page 17 and (112), we have $g(\xi)=\gamma(\xi)=\frac{1}{\hat{\alpha}(\xi v)}$ for any $\xi \in[-\pi, \pi)$. Therefore, we obtain that $g$ is continuous on $([-\pi, \pi])$ and that $g \in$ $L^{1}([-\pi, \pi])$. Since $g \in L^{1}([-\pi, \pi])$ it has a Fourier series decomposition. From the Fourier series decomposition we have

$$
g \underset{p e r}{*} D_{n}(\xi):=\frac{1}{2 \pi} \int_{-\pi}^{\pi} g(y) D_{n}(\xi-y) d y=\sum_{k=-n}^{n} c_{k}(g) e^{i k \xi}
$$

where, for any $k \in \mathbb{Z}$,

$$
c_{k}(g)=\frac{1}{2 \pi} \int_{-\pi}^{\pi} g(\xi) e^{-i k \xi} d x .
$$


We shall now pass to the limit $n \rightarrow+\infty$ in (113). The Fourier series theory entails that $g \underset{\text { per }}{*} D_{n}(\xi):=\frac{1}{2 \pi} \int_{-\pi}^{\pi} g(y) D_{n}(\xi-y) d y \stackrel{n \rightarrow+\infty}{\longrightarrow} g(\xi)$ for any $\xi \in \mathbb{R}$ where $g$ is continuous at $\xi$. Therefore, we obtain that

$$
g(\xi)=\sum_{k=-\infty}^{+\infty} c_{k}(g) e^{i k \xi}
$$

for any $\xi \in \mathbb{R}$ where $g$ is continuous at $\xi$. We have that $g$ is continuous at $\xi=0$. Therefore, from (115) we obtain that

$$
g(0)=\sum_{k=-\infty}^{+\infty} c_{k}(g)
$$

Hence, combining (112), (114) and (116) we deduce that

$$
g(0)=\hat{\gamma}(0)=\sum_{k=-\infty}^{+\infty} \frac{1}{2 \pi} \int_{-\pi}^{\pi} \hat{\gamma}(\xi) e^{-i k \xi} d \xi
$$

Since, from its definition (39) page $17, \gamma$ is $[-\pi, \pi]$ band limited we obtain that

$$
\hat{\gamma}(0)=\sum_{k=-\infty}^{+\infty} \frac{1}{2 \pi} \int_{\mathbb{R}} \hat{\gamma}(\xi) e^{-i k \xi} d \xi
$$

In addition, from (40) we have $\gamma \in L^{2}(\mathbb{R})$. From (xv), for any $k \in \mathbb{Z}$, we have

$$
\frac{1}{2 \pi} \int_{\mathbb{R}} \hat{\gamma}(\xi) e^{-i k \xi} d \xi=\gamma(-k)
$$

Hence, combining (117) and (118) we deduce that

$$
\hat{\gamma}(0)=\int_{\mathbb{R}} \gamma(y) d y=\sum_{k=-\infty}^{+\infty} \gamma(-k) .
$$

This applies for any shift $x \in \mathbb{R}$ of $\gamma$. Hence, from (119) we deduce that

$$
\hat{\gamma}(0)=\int_{\mathbb{R}} \gamma(y) d y=\int_{\mathbb{R}} \gamma(x-y) d y=\sum_{k=-\infty}^{+\infty} \gamma(x-k),
$$

for any $x \in \mathbb{R}$. Thus, combining (41) and (120) we deduce that

$$
\sum_{k=-\infty}^{+\infty} \gamma(x-k)=\frac{1}{\int_{\mathbb{R}} \alpha(x) d x}
$$

for any $x \in \mathbb{R}$. This concludes the proof.

\section{F Proof of lemma 3.6 page 18}

The proof is based on the Poisson summation formula (see annex G page 39). We give the calculations details.

The proof is in two three steps. The step a) proves that, for any $x \in \mathbb{R}$,

$$
\sum_{n=-\infty}^{\infty}\left(\frac{1}{|v|} \alpha\left(\frac{\dot{v}}{v}\right) * \tilde{u}\right)(n) \gamma(x-n)=\left(\left(\frac{1}{|v|} \alpha\left(\frac{\dot{v}}{v}\right) * \tilde{u}\right) * \gamma\right)(x) .
$$


The step $\mathrm{b}$ ) proves that, for any $x \in \mathbb{R}$,

$$
\left(\left(\frac{1}{|v|} \alpha\left(\frac{\cdot}{v}\right) * \tilde{u}\right) * \gamma\right)(x)=\tilde{u}(x) .
$$

The step c) combines the steps a) and b) with lemma 3.5 page 17 and (15) page 13 to deduce (43).

Step a): For any $x \in \mathbb{R}$, we have

$$
\sum_{n=-\infty}^{\infty}\left(\frac{1}{|v|} \alpha\left(\frac{\dot{v}}{v}\right) * \tilde{u}\right)(n) \gamma(x-n)=\int_{-\infty}^{+\infty}\left(\frac{1}{|v|} \alpha\left(\frac{\dot{v}}{v}\right) * \tilde{u}\right)(y) \gamma(x-y) d y .
$$

To justify (123), consider the intermediate function $f_{x}$ defined by

$$
f_{x}(y)=\left(\frac{1}{|v|} \alpha\left(\frac{\dot{v}}{v}\right) * \tilde{u}\right)(y) \gamma(x-y) \text { for any } y \in \mathbb{R} .
$$

With the help of the function $f_{x}$ defined in (124), the left hand side of (123) rewrites as

$$
\sum_{n=-\infty}^{\infty}\left(\frac{1}{|v|} \alpha\left(\frac{\dot{v}}{v}\right) * \tilde{u}\right)(n) \gamma(x-n)=\sum_{n=-\infty}^{\infty} f_{x}(n)
$$

Using the Poisson summation formula (see annex $G$ page 39 ) we will prove that

$$
\sum_{n=-\infty}^{\infty} f_{x}(n) \stackrel{?}{=} \hat{f}_{x}(0)
$$

Provided (126) holds, the equality in (123) follows from the definition of the Fourier transform $\hat{f}_{x}(0)=\int_{\mathbb{R}} f_{x}(y) d y$. Indeed, from the definition (124) of $f_{x}$ we have $\int_{\mathbb{R}} f_{x}(y) d y=\left(\frac{1}{|v|} \alpha(\dot{v}) * \tilde{u}\right)(y) \gamma(x-$ $y) d y$ which coincides with the right hand side of (123). Therefore, provided we prove that (126) holds the equality in (123) holds. We now turn to the validity of (126).

Using the Poisson summation formula (see annex G page 39), we now justify (126). In order to apply the Poisson formula we first need to prove that $f_{x} \in L^{1}(\mathbb{R})$ and band limited. From Cauchy-Schwartz inequality (see, e.g., [25, p. 140]) we have

$$
\left\|f_{x}\right\|_{L^{1}(\mathbb{R})} \leqslant\left\|\frac{1}{|v|} \alpha\left(\frac{\dot{v}}{v}\right) * \tilde{u}\right\|_{L^{2}(\mathbb{R})}\|\gamma\|_{L^{2}(\mathbb{R})} .
$$

In addition, from Young inequality (see, e.g., [25, p. 525]), we have

$$
\left\|\frac{1}{|v|} \alpha\left(\frac{\dot{v}}{v}\right) * \tilde{u}\right\|_{L^{2}(\mathbb{R})} \leqslant\left\|\frac{1}{|v|} \alpha\left(\frac{\dot{v}}{v}\right)\right\|_{L^{2}(\mathbb{R})}\|\tilde{u}\|_{L^{1}(\mathbb{R})} .
$$

Therefore, combining (127) and (128) we have

$$
\left\|f_{x}\right\|_{L^{1}(\mathbb{R})} \leqslant\|\alpha\|_{L^{2}(\mathbb{R})}\|\tilde{u}\|_{L^{1}(\mathbb{R})}\|\gamma\|_{L^{2}(\mathbb{R})} .
$$

Furthermore, from definition 2.1 page 8 we have $\tilde{u} \in L^{1}(\mathbb{R})$, from remark page 11 we have $\alpha \in L^{2}(\mathbb{R})$ and from (40) page 17 we have $\gamma \in L^{2}(\mathbb{R})$. Therefore, from (129) we deduce that $f_{x} \in L^{1}(\mathbb{R})$. From its definition 2.1 page $8 \tilde{u}$ is band limited. Therefore, from its definition (124), 
we deduce that $f_{x}$ is band limited. This means that we can apply the Poisson formula to $f_{x}$. The Poisson summation formula (see annex G page 39) applied to $f_{x}$ entails

$$
\sum_{n=-\infty}^{+\infty} f_{x}(n)=\sum_{m} \hat{f}_{x}(2 m \pi)
$$

Recall that we need to prove that (126) holds. Therefore, we need to prove that the term $m=0$ is the only non zero term in the right hand side of (130). From the definition (124) of $f_{x}$ we have

$$
\hat{f}_{x}(\xi)=\left((\hat{\alpha}(\cdot v) \hat{\tilde{u}}(\cdot)) * \hat{\gamma}(\cdot) e^{-i x \xi}\right)(\xi),
$$

for any $\xi \in \mathbb{R}$. From definition 2.1 page 8 we have that $u$ is $[-\pi, \pi]$ band-limited. This implies that the function $\mathbb{R} \ni \xi \mapsto \hat{\alpha}(\xi v) \hat{\tilde{u}}(\xi)$ is $[-\pi, \pi]$ band limited. From its definition (39) page $17 \gamma$ is also $[-\pi, \pi]$ band-limited. Therefore, from (131), we deduce that $\hat{f}_{x}(\xi)=0$ for all $\xi \in \mathbb{R}$ such that $|\xi|>2 \pi$. Thus, from (130) we deduce that

$$
\sum_{n=-\infty}^{+\infty} f_{x}(n)=\hat{f}_{x}(0)+\hat{f}_{x}(-2 \pi)+\hat{f}_{x}(2 \pi)
$$

Yet, to prove that $(126)$ holds we need to show that $\hat{f}_{x}(-2 \pi)=\hat{f}_{x}(2 \pi)=0$. We now show in details that $\hat{f}_{x}(2 \pi)=0$. From (131), $\hat{f}_{x}(\xi)$ is given by a convolution (see $(\mathbf{x})$ for the definition). Thus, the evaluation of (131) at $\xi=2 \pi$ yields

$$
\hat{f}_{x}(2 \pi)=\int_{\mathbb{R}} \hat{\alpha}(\xi v) \hat{\tilde{u}}(\xi) \hat{\gamma}(2 \pi-\xi) e^{-i x \xi} d \xi
$$

Recall that, from definition 2.1 page $8, \tilde{u}$ is $[-\pi, \pi]$ band-limited. Thus, from (133) we deduce that

$$
f_{x}(2 \pi)=\int_{-\pi}^{\pi} \hat{\alpha}(\xi v) \hat{\tilde{u}}(\xi) \hat{\gamma}(2 \pi-\xi) e^{-i x \xi} d \xi .
$$

The integrand in (134) is non zero only on the zero Lebesque measure set $\{\pi\}$. Hence, we deduce that $\hat{f}_{x}(2 \pi)=0$. Similarly we obtain $\hat{f}_{x}(-2 \pi)=0$. Thus, we proved that (126) holds, i.e., we can remove the question mark in (126). Consequently, we have that (123) holds. Furthermore, from the definition $(\mathrm{x})$ of the convolution we have

$$
\int_{-\infty}^{+\infty}\left(\frac{1}{|v|} \alpha\left(\frac{\dot{v}}{v}\right) * \tilde{u}\right)(y) \gamma(x-y) d y=\left(\left(\frac{1}{|v|} \alpha\left(\frac{\dot{v}}{v}\right) * \tilde{u}\right) * \gamma\right)(x),
$$

for any $x \in \mathbb{R}$. Hence, combining (123) and (135), for any $x \in \mathbb{R}$, we obtain (121) page 36. This concludes the step a). We now turn to step b).

Step b): From the definitions (xv) of the Fourier transform and the inverse Fourier transform, for any $x \in \mathbb{R}$, we have

$$
\left(\left(\frac{1}{|v|} \alpha\left(\frac{\dot{v}}{v}\right) * \tilde{u}\right) * \gamma\right)(x)=\mathcal{F}^{-1}\left(\mathcal{F}\left(\frac{1}{|v|} \alpha\left(\frac{\dot{v}}{v}\right) * \tilde{u}\right)(\xi) \mathcal{F}(\gamma)(\xi)\right)(x) .
$$

Since $\mathcal{F}\left(\frac{1}{|v|} \alpha(\dot{\bar{v}})\right)=\hat{\alpha}(\xi v)$, from (136) we have

$$
\left(\left(\frac{1}{|v|} \alpha(\dot{\bar{v}}) * \tilde{u}\right) * \gamma\right)(x)=\mathcal{F}^{-1}(\hat{\alpha}(\xi v) \hat{\tilde{u}}(\xi) \hat{\gamma}(\xi))(x),
$$


for any $x \in \mathbb{R}$. From the definition of the inverse Fourier transform (xv) and (137) we deduce that, for any $x \in \mathbb{R}$,

$$
\left(\left(\frac{1}{|v|} \alpha\left(\frac{\dot{v}}{v}\right) * \tilde{u}\right) * \gamma\right)(x)=\frac{1}{2 \pi} \int_{-\infty}^{+\infty} \hat{\alpha}(\xi v) \hat{\tilde{u}}(\xi) \hat{\gamma}(\xi) e^{i x \xi} d \xi
$$

From definition 2.1 page 8 we have that $\tilde{u}$ is $[-\pi, \pi]$ band limited. Consequently, from (138) we deduce that, for any $x \in \mathbb{R}$,

$$
\left(\left(\frac{1}{|v|} \alpha\left(\frac{\dot{v}}{v}\right) * \tilde{u}\right) * \gamma\right)(x)=\frac{1}{2 \pi} \int_{-\pi}^{+\pi} \hat{\alpha}(\xi v) \hat{\tilde{u}}(\xi) \hat{\gamma}(\xi) e^{i x \xi} d \xi
$$

From the definition of $\gamma(39)$ page 17 we have that $\hat{\gamma}(\xi)=\frac{1}{\hat{\alpha}(\xi v)}$ for any $\xi \in[-\pi, \pi]$. Hence, we deduce that, for any $x \in \mathbb{R}$,

$$
\left(\left(\frac{1}{|v|} \alpha\left(\frac{\dot{v}}{v}\right) * \tilde{u}\right) * \gamma\right)(x)=\frac{1}{2 \pi} \int_{-\pi}^{\pi} \hat{\tilde{u}}(\xi) e^{i x \xi} d \xi
$$

From definition 2.1 page 8 we have that $\tilde{u}$ is $[-\pi, \pi]$ band limited. This implies that, for any $x \in \mathbb{R}$,

$$
\frac{1}{2 \pi} \int_{\mathbb{R}} \hat{\tilde{u}}(\xi) e^{i x \xi} d \xi=\tilde{u}(x),
$$

where the last equality is justified by the definition of the inverse Fourier transform (xv). Thus, combining (140) and (141) we obtain (122) page 37. This completes the step b). We now turn to the step c) that completes the proof.

Step c): Combining (121) and (122) page 37, for any $x \in \mathbb{R}$, we have

$$
\sum_{n=-\infty}^{\infty}\left(\frac{1}{|v|} \alpha\left(\frac{\dot{v}}{v}\right) * \tilde{u}\right)(n) \gamma(x-n)=\tilde{u}(x) .
$$

From lemma 3.5 page 17 we deduce that, for any $x \in \mathbb{R}$,

$$
\mu\left[\int_{\mathbb{R}} \alpha(t) d t\right] \sum_{n=-\infty}^{+\infty} \gamma(x-n)=\mu .
$$

Therefore, combining (15) page 13, (142) and (143) we deduce that, for any $x \in \mathbb{R}$,

$$
\sum_{n=-\infty}^{\infty}\left(\frac{1}{|v|} \alpha\left(\frac{\dot{v}}{v}\right) * u\right)(n) \gamma(x-n)=\tilde{u}(x)+\mu .
$$

From the definition of $u 2.1$ page 8 we have $u(\cdot)=\tilde{u}(\cdot)+\mu$. Thus, from (144) we obtain that, for any $x \in \mathbb{R},(43)$ page 18 holds. This concludes the proof.

\section{G Poisson summation formula for $L^{1}(\mathbb{R})$ band limited functions}

This section proves that the Poisson summation formula that we use in this paper is valid.

The Poisson summation formula is, formally,

$$
\sum_{n=-\infty}^{+\infty} f(n)=\sum_{m=-\infty}^{+\infty} \hat{f}(2 \pi m) .
$$


However, (145) is not always valid when $f \in L^{1}(\mathbb{R})$. Indeed, even when both sides of (145) converge absolutely the equality may fail (see, e.g., [13] and [21, Ex 1.17, p. 163] for an example of $f \in L^{1}(\mathbb{R})$ such that $\hat{f} \in L^{1}(\mathbb{R})$ for which (145) fails).

A classic condition that ensures that (145) holds involves decay estimates of both $f$ and $\hat{f}$ at the infinity (see, e.g., [25, thm. 1, p. 628]) and does not apply if $f$ is just in $L^{1}(\mathbb{R})$ and band limited. Other results involve bounded variation assumption (see, e.g., [13]) on $f$ is not applicable here. Therefore, we shall now provide a proof that (145) holds in our case that is for an $f \in L^{1}(\mathbb{R})$ and band limited. Our proof is based on [8].

Let $f \in L^{1}(\mathbb{R})$ be such that $\hat{f}(\xi)=0$ for any $\xi$ such that $|\xi|>C$ for some constant $C$. From Riemann-Lebesgue theorem (see e.g., [23, prop. 2.1]) we have $\hat{f}$ is continuous. From the band limited assumption we deduce that $\hat{f} \in L^{1}(\mathbb{R})$. Since $f$ is band limited the series $\sum_{m=-\infty}^{+\infty} \hat{f}(2 \pi m-x)$ is finite and therefore converges uniformly in $x \in \mathbb{R}$. By Plancherel-Pólya inequality (see [49, equations (25) and (26), p. 233], since $f \in L^{1}(\mathbb{R})$ and band limited it satisfies the growth condition) we obtain that $\sum_{k=-\infty}^{+\infty}|f(k)| \leqslant A\|f\|_{L^{1}(\mathbb{R})}$ for some positive constant $A$. Therefore, the series $\sum_{k=-\infty}^{+\infty} f(k)$ converges. Thus, we can apply [8, theorem 2, p.147] (with $g:=\hat{f}$ and $W=\frac{1}{2 \pi}$ ) to deduce that (145) is valid for any $f \in L^{1}(\mathbb{R})$ band limited.

\section{H Main notations and formulae}

(i) $t \geqslant 0$ time variable

(ii) $\Delta t$ length of a time interval (exposure time)

(iii) $x \in \mathbb{R}$ spatial variable

(iv) $\bar{z}$, for $z \in \mathbb{C}$ denotes the conjugate of a complex number

(v) $X \sim Y$ means that the random variables $X$ and $Y$ have the same law

(vi) $\mathbb{P}(A)$ probability of an event $A$

(vii) $\mathbb{E}(X)$ expected value of a random variable $X$

(viii) $\operatorname{var}(X)$ variance of a random variable $X$

(ix) $\mathcal{P}(\lambda)$ Poisson random variable with intensity $\lambda>0$. Thus, if $X \sim \mathcal{P}(\lambda)$ we have $\mathbb{P}(X=$ $k)=\frac{\exp (-\lambda) \lambda^{k}}{k !}$

(x) $f * g$ convolution of two functions $(f * g)(x)=\int_{\mathbb{R}} f(y) g(x-y) d y$

(xi) obs(n), $n \in \mathbb{Z}$ observation of the scene at pixel $n$

(xii) $v$ relative velocity between the scene and the camera (unit: pixel(s) per second)

(xiii) $\alpha(\cdot)=\sum_{k=-\infty}^{+\infty} \alpha_{k} \mathbb{1}_{[k \Delta t,(k+1) \Delta t[}(\cdot)$ exposure function $\left(\left(\alpha_{k}\right)_{k} \in \ell^{1}(\mathbb{Z})\right)$

(xiv) $\|f\|_{L^{1}(\mathbb{R})}=\int_{\mathbb{R}}|f(x)| d x,\|f\|_{L^{2}(\mathbb{R})}=\sqrt{\int_{\mathbb{R}}|f(x)|^{2} d x}$

(xv) Let $f, g \in L^{1}(\mathbb{R})$ or $L^{2}(\mathbb{R})$, then $\mathcal{F}(f)(\xi):=\hat{f}(\xi):=\int_{\mathbb{R}} f(x) e^{-i x \xi} d x$ and $\mathcal{F}^{-1}(\mathcal{F}(f))(x):=\overline{\mathcal{F}(f)}(x)=f(x)=\frac{1}{2 \pi} \int_{\mathbb{R}} \mathcal{F}(f)(\xi) e^{i x \xi} d \xi$. Moreover $\mathcal{F}(f * g)(\xi)=\mathcal{F}(f)(\xi) \mathcal{F}(g)(\xi)$ and (Plancherel)

$$
\int_{\mathbb{R}}|f(x)|^{2} d x=\|f\|_{L^{2}(\mathbb{R})}^{2}=\frac{1}{2 \pi} \int_{\mathbb{R}}|\mathcal{F}(f)|^{2}(\xi) d \xi=\frac{1}{2 \pi}\|\mathcal{F}(f)\|_{L^{2}(\mathbb{R})}^{2}
$$


(xvi) $u$ ideal (noiseless) observable scene.

$\left(\right.$ xvii) $\operatorname{sinc}(x)=\frac{\sin (\pi x)}{\pi x}=\frac{1}{2 \pi} \mathcal{F}\left(\mathbb{1}_{[-\pi, \pi]}\right)(x)=\mathcal{F}^{-1}\left(\mathbb{1}_{[-\pi, \pi]}\right)(x)$

(xviii) $\mathbb{1}_{[a, b]}$ indicator function of an interval $[a, b]$

(xix) Let $f \in L^{1}([-R, R])$ or $f \in L^{2}([-R, R])$. The $n$-th Fourier series coefficient of $f$ is $c_{n}(f):=\frac{1}{2 R} \int_{-R}^{R} f(t) e^{-i n t \frac{\pi}{T}} d t$ and we have $f(t)=\sum_{n=-\infty}^{+\infty} c_{n}(f) e^{+i n t \frac{\pi}{T}}$. Moreover we have (Parseval)

$$
\sum_{n=-\infty}^{+\infty}\left|c_{n}(f)\right|^{2}=\frac{1}{2 R} \int_{-R}^{R}|f(t)|^{2} d t
$$

\section{References}

[1] A. Agrawal, M. Gupta, A. Veeraraghavan, and S.G. Narasimhan, Optimal coded sampling for temporal super-resolution, in the Proceedings of the IEEE Conference on Computer Vision and Pattern Recognition (CVPR), 2010, pp. 599-606.

[2] A. Agrawal And R. Raskar, Resolving objects at higher resolution from a single motionblurred image, in the Proceedings of the IEEE Conference on Computer Vision and Pattern Recognition (CVPR), 2007, pp. 1-8.

[3] — Optimal single image capture for motion deblurring, in the Proceedings of the IEEE Conference on Computer Vision and Pattern Recognition (CVPR), 2009, pp. 2560-2567.

[4] A. Agrawal and Y. Xu, Coded exposure deblurring: Optimized codes for PSF estimation and invertibility, in the Proceedings of the IEEE Conference on Computer Vision and Pattern Recognition (CVPR), 2009, pp. 2066-2073.

[5] A. Agrawal, Y. Xu, and R. Raskar, Invertible motion blur in video, ACM Transactions on Graphics (TOG), 28 (2009), pp. 95:1-95:8.

[6] J.M. Bony, Cours d'analyse: théorie des distributions et analyse de Fourier, Éditions de l'École Polytechnique, 2001.

[7] G. Boracchi And A. FoI, Uniform Motion Blur in Poissonian Noise: Blur/Noise Tradeoff, IEEE Transactions on Image Processing, 20 (2011), pp. 592-598.

[8] P.L. Butzer And R.L. Stens, The Euler-MacLaurin summation formula, the sampling theorem, and approximate integration over the real axis, Linear Algebra and its Applications, 52 (1983), pp. 141-155.

[9] T. Chonavel and J. Ormrod, Statistical Signal Processing: Modelling and Estimation, Advanced Textbooks in Control and Signal Processing, Springer London, 2002.

[10] R. Dahlhaus, Mathematical Methods in Signal Processing and Digital Image Analysis, Springer complexity, Springer, 2008.

[11] L. Dengyu, G. Jinwei, Y. Hitomi, M. Gupta, T. Mitsunaga, and S.K. Nayar, Efficient Space-Time Sampling with Pixel-Wise Coded Exposure for High-Speed Imaging, IEEE Transactions on Pattern Analysis and Machine Intelligence, 36 (2014), pp. 248-260. 
[12] Y. Ding, S. McCloskey, And J. Yu, Analysis of motion blur with a flutter shutter camera for non-linear motion, in the Proceedings of the Springer-Verlag European Conference on Computer Vision (ECCV), 2010, pp. 15-30.

[13] A.L. Durán, R. Estrada, and R.P. Kanwal, Extensions of the Poisson summation formula, Journal of mathematical analysis and applications, 218 (1998), pp. 581-606.

[14] D. GaO, D. Liu, X. XIE, X. Wu, And G. Shi, High-resolution multispectral imaging with random coded exposure, Journal of Applied Remote Sensing, 7 (2013), pp. 73695-73695.

[15] C. Gasquet and P. Witomski, Fourier Analysis and Applications: Filtering, Numerical Computation, Wavelets, Texts in Applied Mathematics, U.S. Government Printing Office, 1999.

[16] J. Holloway, A. C. Sankaranarayanan, A. Veeraraghavan, and S. Tambe, Flutter shutter video camera for compressive sensing of videos, in the Proceedings of the IEEE International Conference on Computational Photography (ICCP), 2012, pp. 1-9.

[17] K. Huang, J. Zhang, and G. Li, Noise-optimal capture for coded exposure photography, Optical Engineering, 51 (2012), pp. 093202-1-093202-6.

[18] J. JELINEK, Designing the optimal shutter sequences for the flutter shutter imaging method, in SPIE Defense, Security, and Sensing, International Society for Optics and Photonics, 2010, pp. $77010 \mathrm{~N}-77010 \mathrm{~N}$.

[19] J. Jelinek And S. MCCloskey, Method and system for designing optimal flutter shutter sequence, Sept. 17 2013. US Patent 8,537,272.

[20] A. F. KARR, Probability, Springer Texts in Statistics, 1993.

[21] Y. Katznelson, An Introduction to Harmonic Analysis, Cambridge Mathematical Library, Cambridge University Press, 2004.

[22] B. Kovacevic, Z. Durovic, And Z. Durović, Fundamentals of Stochastic Signals, Systems and Estimation Theory: With Worked Examples, Academic Mind, 2008.

[23] S.G. Krantz, A Panorama of Harmonic Analysis, American History Series, Mathematical Association of America, 1999.

[24] R. Lukac, Computational Photography: Methods and Applications, vol. 2, CRC Press, 2010 .

[25] B. Makarov and A. Podkorytov, Real Analysis: Measures, Integrals and Applications, Universitext, Springer, 2013.

[26] R.J. MARKS, Introduction to Shannon sampling and interpolation theory, Springer texts in electrical engineering, Springer-Verlag, 1991.

[27] S. MCCloskey, Velocity-dependent shutter sequences for motion deblurring, in the Proceedings of the Springer-Verlag European Conference on Computer Vision (ECCV), 2010, pp. 309-322.

[28] S. Mccloskey, Acquisition system for obtaining sharp barcode images despite motion, Feb. 16 2011. EP Patent 2,284,764. 
[29] S. McCloskey, Temporally coded flash illumination for motion deblurring, in the Proceedings of the IEEE International Conference on Computer Vision (ICCV), 2011, pp. 683-690.

[30] — Fluttering illumination system and method for encoding the appearance of a moving object, Oct. 23 2012. US Patent 8,294,775.

[31] — - Heterogeneous video capturing system, May 7 2013. US Patent 8,436,907.

[32] —, Motion Deblurring: Algorithms and Systems, Cambridge University Press, 2014, ch. 11: Coded exposure motion deblurring for recognition.

[33] S. McCloskey, W. AU, AND J. JelineK, Iris capture from moving subjects using a fluttering shutter, in the Proceedings of the IEEE International Conference on Biometrics: Theory Applications and Systems (BTAS), 2010, pp. 1-6.

[34] S. McCloskey, Y. Ding, And J. Yu, Design and Estimation of Coded Exposure Point Spread Functions, IEEE Transactions on Pattern Analysis and Machine Intelligence, 34 (2012), pp. 2071-2077.

[35] S. McCloskey, J. Jelinek, And K.W. Au, Method and system for determining shutter fluttering sequence, Apr. 9 2009. US Patent 12/421,296.

[36] S. McCloskey, K. Muldoon, and S. Venkatesha, Motion invariance and custom blur from lens motion, in the Proceedings of the IEEE International Conference on Computational Photography (ICCP), 2011, pp. 1-8.

[37] R. RASKAR, Method and apparatus for deblurring images, July 13 2010. US Patent $7,756,407$.

[38] R. Raskar, A. Agrawal, and J. Tumblin, Coded exposure photography: motion deblurring using fluttered shutter, ACM Transactions on Graphics (TOG), 25 (2006), pp. 795-804.

[39] R. Raskar, J. Tumblin, and A. Agrawal, Method for deblurring images using optimized temporal coding patterns, Aug. 25 2009. US Patent 7,580,620.

[40] D. Reddy, A. Veeraraghavan, and R. Raskar, Coded Strobing Photography for HighSpeed Periodic Events, in Imaging Systems, Optical Society of America, 2010.

[41] S. Resnick, A Probability Path, Birkhäuser Boston, 1999.

[42] A. Sarker And L.G.C. Hamey, Improved Reconstruction of Flutter Shutter Images for Motion Blur Reduction, in the Proceedings of the IEEE International Conference on Digital Image Computing: Techniques and Applications (DICTA), 2010, pp. 417-422.

[43] E. F. Schonbrun, S. S. Gorthi, And D. SchaAk, Fluorescence flutter shutter for imaging cells in flow, in Computational Optical Sensing and Imaging, Optical Society of America, 2013, pp. CTh4B-4.

[44] A.N. ShiRyaev, Probability, Graduate texts in mathematics, Springer-Verlag, 1984.

[45] E.M. Stein And G.L. Weiss, Introduction to Fourier Analysis on Euclidean Spaces, Mathematical Series, Princeton University Press, 1971.

[46] Y.W. TaI, N. Kong, S. Lin, And S.Y. Shin, Coded exposure imaging for projective motion deblurring, in the Proceedings of the IEEE Conference on Computer Vision and Pattern Recognition (CVPR), 2010, pp. 2408-2415. 
[47] Y. Tendero, The Flutter Shutter Camera Simulator, Image Processing On Line, 2012 (2012).

[48] Y. Tendero, J.-M. Morel, And B. Rougé, The Flutter Shutter Paradox, SIAM Journal on Imaging Sciences, 6 (2013), pp. 813-847.

[49] A.F. Timan, Theory of Approximation of Functions of a Real Variable, Dover books on advanced mathematics, Pergamon Press, 1963.

[50] S. Toril And Y. SHIndo, Information processing apparatus and method for synthesizing corrected image data, Feb. 19 2013. US Patent 8,379,096.

[51] R. Tsai, Pulsed control of camera flash, June 14 2011. US Patent 7,962,031.

[52] S. Tsutsumi, Image processing apparatus and method, 2011. US Patent App. 13/104,476.

[53] A. Veeraraghavan, D. Reddy, and R. Raskar, Coded Strobing Photography: Compressive Sensing of High Speed Periodic Events, IEEE Transactions on Pattern Analysis and Machine Intelligence, 33 (2011), pp. 671-686.

[54] W. Xu And S. MCCloskey, 2D Barcode localization and motion deblurring using a flutter shutter camera, in the Proceedings of the IEEE Workshop on Applications of Computer Vision (WACV), 2011, pp. 159-165.

[55] A. Zaknich, Principles of Adaptive Filters and Self-learning Systems, Advanced Textbooks in Control and Signal Processing, Springer London, 2006. 